\title{
Identification and Toxicological Assessment of Thermal Degradation Products of Organic Constituents of Parts Comprising LLNL Designs ARG Phase 2 Interim Report
}

A.E. Lipska-Quinn and R.D. Lopez

U.S. Department of Energy

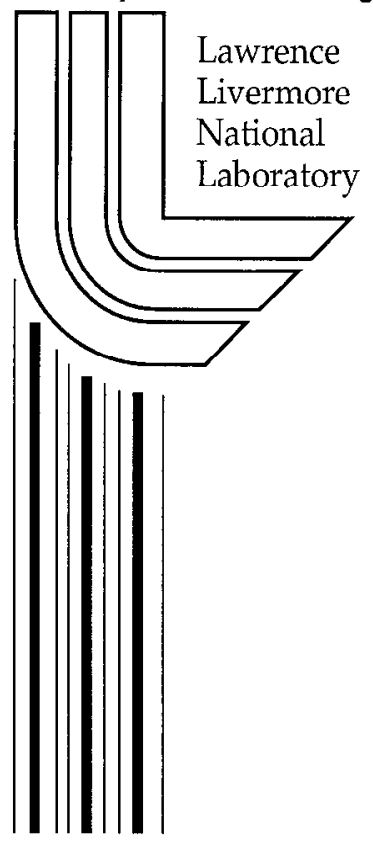

October 28, 1999 


\section{DISCLAIMER}

This document was prepared as an account of work sponsored by an agency of the United States Government. Neither the United States Government nor the University of California nor any of their employees, makes any warranty, express or implied, or assumes any legal liability or responsibility for the accuracy, completeness, or usefulness of any information, apparatus, product, or process disclosed, or represents that its use would not infringe privately owned rights. Reference herein to any specific commercial product, process, or service by trade name, trademark, manufacturer, or otherwise, does not necessarily constitute or imply its endorsement, recommendation, or favoring by the United States Government or the University of California. The views and opinions of authors expressed herein do not necessarily state or reflect those of the United States Government or the University of California, and shall not be used for advertising or product endorsement purposes.

Work performed under the auspices of the U. S. Department of Energy by the University of California Lawrence Livermore National Laboratory under Contract W-7405-Eng-48.

This report has been reproduced directly from the best available copy.

Available to DOE and DOE contractors from the

Office of Scientific and Technical Information

P.O. Box 62, Oak Ridge, TN 37831

Prices available from (423) 576-8401

http://apollo.osti.gov/bridge/

Available to the public from the National Technical Information Service

U.S. Department of Commerce 5285 Port Royal Rd., Springfield, VA 22161 http://www.ntis.gov/

OR

Lawrence Livermore National Laboratory Technical Information Department's Digital Library http://www.llnl.gov/tid/Library.html 
Identification and Toxicological Assessment of Thermal Degradation Products of Organic Constituents of Parts Comprising LLNL Designs

Anne E. Lipska-Quinn and Ronald D. Lopez

\section{PURPOSE}

The goals of Phase II of the Non Rad Toxics Project were:

1. To identify and quantify the major chemicals comprising smoke from sinuldering plastics most prevalent in the LINL designed weapons as well as materials unique to LANL designed weapons and SANDIA parts sound in the LLNL and LANL weapons.

2. To perfurmi !oxic assessment of the identified chemicals vising existing literature information or TOPKAT, a computer program designed for toxic assessment of organic and certain organo-metallic compourids. This project was in support of the ARG Program needs \#15, Table XI, Accident Response Group.

\section{APPROACH}

The plastics chosen for this study were obtained largely from PANTEX. Some of the materials were individual pieces of plastics such as polyurethane, polycarbonate, or cellular silicone, while others were small pieces of various cable insulations which consisted of several layers of different types of plastics. This was done to determine whether smoke generated from plastic composites, a more realistic scenario in an accident, would contain a more complex and/or riore toxic chemical mixture.

The assessment of the toxic nature of the major components in the smoke was done with the computer program TOPKAT. This program estimates toxic effects of chemical structures using statistical quantitative structure activity relationships (QSAR) terhniques, including discriminant and regression analysis.

\section{EXPERIMENTAL}

\section{Sample Preparation}

Finely divided samples weighing $1 \mathrm{~g}$. each were placed in a glass pyrolyzer which was enclosed in a tube oven capable of being heated up to $1000^{\circ} \mathrm{C}$. Samples were placed in the center of the pyrolyzer and heated to 
temperature resembling that of smoldering conditions for a period of time needed to completely degrade the samples. One end of the pyrolyzer was connected to an air/inert gas inlet, which supplied a gentle stream of air or nitrogen during the course of sample heating as well as swept the generated smoke into a cold trap, which was connected, to the pyrolyzer at its exit. The cold trap was placed in a Dewar filled with liquid nitrogen. At the end of pyrolysis, the cold trap was brought to room temperature and the liquid contents were diluted with $1 \mathrm{ml}$ of ethylene chloride. Prior to analysis, 10 microliters of $\mathrm{n}$-decane (an internal standard) were added to the mixture.

Sample Analysis

The mixture was analyzed via a Hewlett-Packard gas chromatograph equipped with a mass spectrometer. Two microliters of the sample were injected on a fused-silica capillary column (J\&W, DB1, bonded phase, $0.25 \mu \mathrm{m}$ phase thickness, $30 \mathrm{~m} \times 02 \mathrm{~mm}$ ). The inlet mode was spiltless with an injector's temperature of $250^{\circ} \mathrm{C}$. A programmed temperature setting were as follows: initial temperature $50^{\circ} \mathrm{C}$, hold $5 \mathrm{~min}$, increase at $5^{\circ} \mathrm{C} / \mathrm{min}$ to $250^{\circ} \mathrm{C}$ and hold $20 \mathrm{~min}$.

The separated components were identified with the mass spectrometer by comparing the fragmentation patterns of the unknown compounds to those of known compounds compiled in a spectral library system.

Toxic assessment of the major components comprising the various mixtures was done with TOPKAT. The major areas considered were: carcinogenicity, developmental toxicity potential (DTP), mutagenicity (AMES), Rat Oral LD50, Rat Chronic Lowest Observed Adverse Effect (LOEL), Rabbit Eye Irritation and Rabbit Skin Irritation.

\section{RESULTS}

Results are tabulated in terms of chromatograms and Excel spreadsheets. The spreadsheets list the name of the main liquid chemicals their amounts (nanograms/microliter and their toxic assessment. The abbreviation CBE is used when values could not be estimated and IND refers to indeterminate values. The six modules used in the toxic assessment studies are summarized as follows:

Rodent Carcinogenicity

The Rodent Carcinogenicity Module comprises four statistically significant and cross-validated quantitative structure-toxicity relationship (QSTR) models, and the data from which the models are derived. Sub-models have been developed for each sex/species: Male Rat, Female Rat, Male Mouse, and Female Mouse. Molecular structure is the only input required to conduct a carcinogenicity assessment. These discriminate models compute the probability of a submitted chemical structure being a carcinogen in male and female rats and male and female mice. 
Toxicity values are computed by summing the individual contributions for assessing toxicity values such as $\mathrm{LD}_{50}$ or $\mathrm{LC}_{50^{\circ}}$. This sum is transformed into a weight/weight unit $(\mathrm{mg} / \mathrm{kg}$ ). For two group classifications, such as carcinogens/ noncarcinogens this sum is transformed into a probability value between 0.0 to 0.30 and is considered low or negative probabilities. Probabilities greater than 0.30 , but less than 0.70 , are considered to be a indeterminate (IND) response in an experimental assay, whereas probabilitý values greater than 0.70 are considered high, and are likely to wroduce a highly positive response in an experimental assay.

\section{DTP}

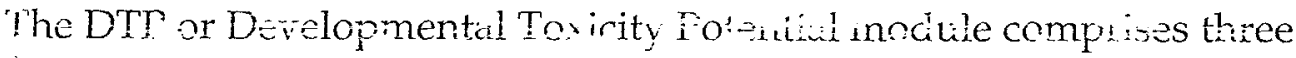
staristicaliy significant and coss valicied quant tetive s-ucture-tcoicity relaticn

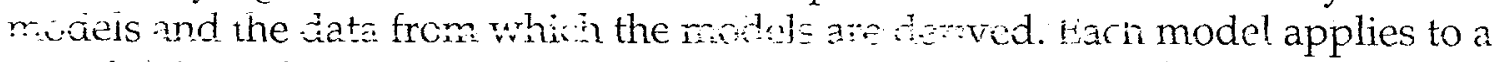

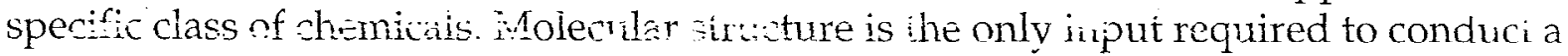
Dir' assessment. These discriminant models compute the probability of a submittod chemical structure being a developmental toxicant in the rat. A probability betwee: 0.1 and 0.29 indicate a low potential for developmental toxicity. The probability range between 0.3 and 0.7 refers to indeterminant (IND). The probability range from 0.7 to 1.0 signifies a high potential for developmental toxicity. These models are derived from 273 experimental studies extracted from approximately 3,000 open literature sources.

\section{(AMES) Mutagenicity}

The Ames Mutagenicity Module comprises 10 statistically significant and cross- validated quantitative structure - toxicity relationship (QTSR) models, and the data from which the models are derived. Each model applies to a specific class of chemicals. Molecular structure is the only input required to conduct a mutagenicity assessment. These discriminant models compute the probability of a submilted chemical structure being a mutagen in the histidine reversion assay; a probability below 0.29 indicates a non-mutagen (NEG), (NO), and that above 0.7 signifies a mutagen (POS), (YES). The probability range between 0.3 and 0.7 refers to the indeterminate zone (IND). These models are derived from the results of 1866 histidine reversion assays using Salmoneila typhimurium stiains. Experimentally, a chemical is considered NEG only if it does not show any mutagenic activity in all tested strains.

\section{Rat Oral LD 50}

The Rat Oral $\mathrm{LD}_{50}$ module comprises 19 statistically significant and cross validated quantitative structure-toxicity relationship (QSTR) models, and the data from which the models are derived. Each QSTR model assesses oral acute median 
lethal dose, $\mathrm{LD}_{50}$ in the rat of a specific class of chemicals. Molecular structure is the only input required to conduct an $\mathrm{LD}_{50}$ assessment. These models are derived from experimental $\mathrm{LD}_{50}$ values of approximately 4,000 chemicals.

\section{Rat Chronic LOAEL}

The Rat Chronic Lowest Observed Adverse Effect (LOAEL) module comprises statistically significant and cross-validated quantitative structure-toxicity relationship (QSTR) models, and the data from which the models are derived. These models are derived irom experimental LOAEL values of approximately 393 chemicals. Lach QSTR model assesses the lowest observed adverse effect level in the rat from oral

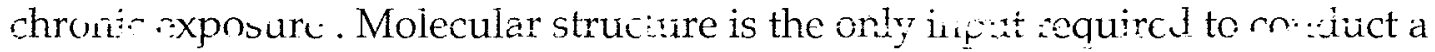
LC:EI arnossment.

Eabb: iye Irritation

Or

Rābbił Skin Irritation

This model estimates the severity of eye or skin irritation if a rabbit eye or skin irritation test were performed on the compound of interest. Separate equations are used for compounds with rings and compounds without rings. For compounds with rings there are two submodels: the first separates severes from all others; the second scparates moderate/severes from negative/milds. For compounds without rings, the first submodel separates severes from all others, and the second separates negatives from all others. The results from the two submodels are used in a decision table to produce the composite estimate of eye or skin irritation severity.

Excel spreadsheets show that all the plastics form smoke during smoldcring that contains chemicals that are skin and eye irritants. Of these, a number are categorized as severe eye and skin irritants. The TOPKAT analysis also shows that smoke from the various plastics contains considerable amounts of carcinogens and some mutagens. Plastics responsible for production of chemicals identified as having moderate or high developmental toxicity potential are polystyrene, nomex, polyurethane and cellular silicone. The combination of polyurethane, nylon, and polyvinyledene fluoride yields a mixture with greater number of chemicals witli high developmental toxicity potential. On the whole most of the chemicals identified exhibit reasonably high Rat Oral $L_{50}$. The Rat Chronic Lowest Observed Adverse Effect in most cases requircs exposure of the rat to chemicals at reasonably high concentrations. Exception to this observation is shown in $1.3 \mathrm{mg} / \mathrm{kg}$ of cyclopropanecarboxylic acid, 2,2-dimethyl-3-2 formed from viton, $3.1 \mathrm{mg} / \mathrm{kg}$ of 2.3 dihydro-benzofuran and $4.1 \mathrm{mg} / \mathrm{kg}$ pyrazine, methyl-4 oxide both formed from polycarbonate, $1.5 \mathrm{mg} / \mathrm{kg} 1.1^{\prime}$-ethenylidene bis-benzene from polystyrene, $4.7 \mathrm{mg} / \mathrm{kg}$ 2-pentanone,3-methyl from neoprene and teflon insulation. 


\section{CONCLUSIONS AND RECOMMENDATIONS FOR FUTURE WORK}

Results of this preliminary study show that smoke from all plastics investigated contains some chemicals that are carcinogens, mutagens, as well as eye and skin irritants. Results also suggest that a smoldering pile of several different kinds of plastics will lead to production of smoke that will contain more complex and more toxic mixture of chemicals. This study should be extended to other plastics tiiat due to lack $c_{i}$ funds were omitted. In addition. these results should bc compared with findings from measurements done on smoke

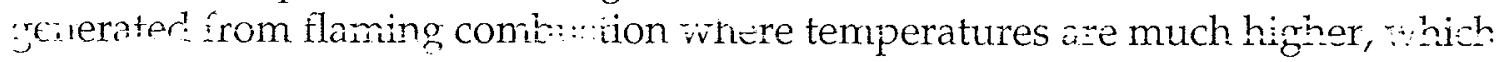
in tur: in aty lead to different tvpe of rhemical mixtures. 


\begin{tabular}{|c|c|c|c|c|c|c|c|c|c|c|c|c|}
\hline Chemical & $\begin{array}{c}\text { Rotantion } \\
\text { Throe }\end{array}$ & & Carcin & genic & city & $\begin{array}{c}\text { AMES } \\
\text { Mutagenicity }\end{array}$ & Rat Oral & Irritatio & Model & $\begin{array}{l}\text { Developmental } \\
\text { Toxicity }\end{array}$ & Chronic LOAEL & Amount ng \\
\hline Compound & $($ min $)$ & $\begin{array}{l}\text { Fombi } \\
\text { Mouse }\end{array}$ & Female & Mouse & $\begin{array}{l}\text { Molo } \\
\text { Rat }\end{array}$ & & Lo so & Eye & Skin & & & \\
\hline Cyclopropanecarboxylic acid,2-22-dimethyt-3-2. & 24 & HEG & $\mathrm{HHGH}$ & NO & HGH & No & $109 \mathrm{~kg}$ & MLD & MODERATE & LOW & $14 \mathrm{mg} \mathrm{kg}$ & 119.3 \\
\hline Ethanone, 1 [4-(1-hydroxy-1-methylethyl)pheny $]$ & 27.2 & Low & NO & Low & Low & LOW & $2.7 \mathrm{~g} / \mathrm{kg}$ & MOOERATE & MODERATE & NO & $3.5 \mathrm{~g} \mathbf{k g}$ & 2.2 \\
\hline i-propanone, 3 -cyclopentyl-1-(2,4-dimethylphenyl & 28.7 & HWG & Low & NO & LOW & MGH & $965 \mathrm{mg} / \mathrm{kg}$ & MID & MODERATE & HIGH & $3.1 \mathrm{~g} / \mathrm{kg}$ & 8.3 \\
\hline Pheno, 2,4-bls (1-methylethy! & 29.7 & $\mathrm{HHGH}$ & NO & NO & MODERATE & NO & $2.4 \mathrm{~g} / \mathrm{kg}$ & MLD & SEVERE & NO & $388 \mathrm{mg} / \mathrm{kg}$ & 0.875 \\
\hline Naphtalene, i,6-dimethyl-4-(i-methylethyl & 33.5 & HGG & HIGH & $\mathrm{NO}$ & MODERATE & HGH & $3.2 \mathrm{~g} / \mathrm{kg}$ & MODERATE & SEVERE & Low & $26 \mathrm{mg} k g$ & 0.61 \\
\hline Carbonic acid, 4-methylphenyl phenyl ester & 33.8 & Low & MGH & HGH & LOW & HGG & $2.5 \mathrm{~g} / \mathrm{kg}$ & SEVERE & MODERATE & MOOERATE & $28 \mathrm{mg} / \mathrm{kg}$ & 0.73 \\
\hline Benzene, I-methyl-2-I(3-methylphenyi)methy]] & 34.4 & HGG & HIGH & NO & LOW & NO & $2 g / \mathrm{kg}$ & MODERATE & MOOERATE & MIGH & $47 \mathrm{mg} \mathrm{kg}$ & $0 . \overline{6} \mathbf{1}$ \\
\hline Benzoic acid,4-hydroxy-3,5-dimethoxy-,methy! & 34.8 & Low & NO & Low & $\mathrm{HGH}$ & LOW & $4 \mathrm{~g} \mathbf{k g}$ & SEVERE & MOOERATE & $\mathrm{HGH}$ & $1.3 \mathrm{~g} \mathbf{k g}$ & 18 \\
\hline Pheno, 4,4'-methylenebis-Scajed & 39 & No & HIGH & HGH & HGG & MODERATE & $511 \mathrm{mg} / \mathrm{kg}$ & MOOERATE & NO & NO & $118 \mathrm{mg} k g$ & 0.58 \\
\hline Phend,4,4-(1-methyletnylidene) bis & 41 & $\overline{10}$ & Low & LOW & NO & Low & $2.7 \mathrm{q} / \mathrm{kg}$ & SEVERE & MOOERATE & NO & $65 \mathrm{mg} / \mathrm{kg}$ & 0.62 \\
\hline Phenol, $2-[1,-(4-h y d r o x y p h e n y l)-1-$ methylethyi $]$ & 42 & Low & NO & NO & NO & NO & $2.5 \mathrm{~g} / \mathrm{kg}$ & SEVERE & MOOERATE & NO & $40 \mathrm{mg} / \mathbf{k g}$ & $0.1 ?$ \\
\hline$[1,1-B i p h e n y)]-2-0,5-(1,1$ dimethylethy $)$ & 42.6 & NO & Low & Low & LOW & $\bar{w}$ & $4.7 \mathrm{~g} / \mathrm{kg}$ & SEVERE & MODERATE & Low & $54 \mathrm{mg} \cdot \mathrm{kg}$ & 0.02 \\
\hline 1-Pentano $2,2,3,3,4,4,5,5$-octaftuoro & 4.2 & HGH & NO & HGH & NO & NO & $755 \mathrm{mg} / \mathrm{kg}$ & MODERATE & MODERATE & NO & $131 \mathrm{mg} \mathrm{kg}$ & 2.75 \\
\hline 3-Decene,2,2-dimethyt-(E) & 4.9 & HGH & NO & No & NO & HGH & $10 \mathrm{~g} / \mathrm{kg}$ & MODERATE & MOOERATE & NO & $839 \mathrm{mg} / \mathrm{kg}$ & 2.1 \\
\hline $2,6,10$-0odecatrienoic acid, $3,7,11$-trimethyl & $5 . \overline{8}$ & HIGH & HIGH & HGH & LOW & MOOERATE & $1.3 \mathrm{~g} / \mathrm{kg}$ & MOOERATE & MODERATE & NO & $4.2 \mathrm{mg} / \mathrm{kg}$ & 12.6 \\
\hline Benzoic acid,2-(4-metnylbenzoyl)-, methy! ester & 5.9 & $H G H$ & NO & HIGH & MODERATE & $\mathrm{HGH}$ & $2.9 \mathrm{~g} / \mathrm{kg}$ & ND & SEVERE & Low & $130 \mathrm{mg} / \mathrm{kg}$ & 3.7 \\
\hline Benzene, 1-(1,1-dimethylethyli)-4-ethoxy & 6.6 & NO & HIGH & $\mathrm{HGH}$ & LOW & NO & $3.1 \mathrm{~g} / \mathrm{kg}$ & SEVERE & MOOERATE & Low & $219 \mathrm{mg} \mathrm{kg}$ & -4.5 \\
\hline 1, Propene, $1,1,3,3,3$ - pentafluoro & 8.7 & $\overline{H G H}$ & NO & NO & HGH & NO & $30 / k g$ & SEVERE & MOOERATE & Low & $87 \mathrm{mg} / \mathrm{kg}$ & 3.7 \\
\hline Pentane, $1,1,2,2,3,3,4,4$-octafluoro & 10 & HGH & NO & HIGH. & NO & NO & $19 \mathrm{~kg}$ & MODERATE & MODERATE & NO & $139 \mathrm{mg} / \mathrm{kg}$ & 35.8 \\
\hline Nonane,3-methÿl $\cdots$ & 10.5 & HEH & NO & NO & HGGH & NO & $109 / \mathrm{kg}$ & MODERATE & MODERATE & NO & $7 \mathrm{mg} / \overline{\mathrm{kg}}$ & 8.5 \\
\hline 2. Propenoic acid, 2-methyl-1,2- ethaneditlbis & 10.6 & NO & LOW & $\overline{\mathrm{NO}}$ & HGH & NO & $10 \mathrm{~g} / \mathrm{kg}$ & SEVERE & MODERATE & NO & $109 \times 9$ & 2.65 \\
\hline Phthahc antydride & 21.2 & LOW & NO & Low & Low & NO & $1.5 \mathrm{~g} / \mathrm{kg}$ & SEVERE & MLO & LOW & $266 \mathrm{mg} / \mathrm{kg}$ & 8.5 \\
\hline Cyclopropanecarboxylic acid,2,2- dimethyl-3-2 & 21.4 & NO & HIGH & NO & HGH & Lố- & $5.8 \mathrm{~g} / \mathrm{kg}$ & MLD & MODERATE & NO & $1.3 \mathrm{mg} / \mathrm{kg}$ & 3.7 \\
\hline
\end{tabular}


TIC of vitonS1.d

Abundance

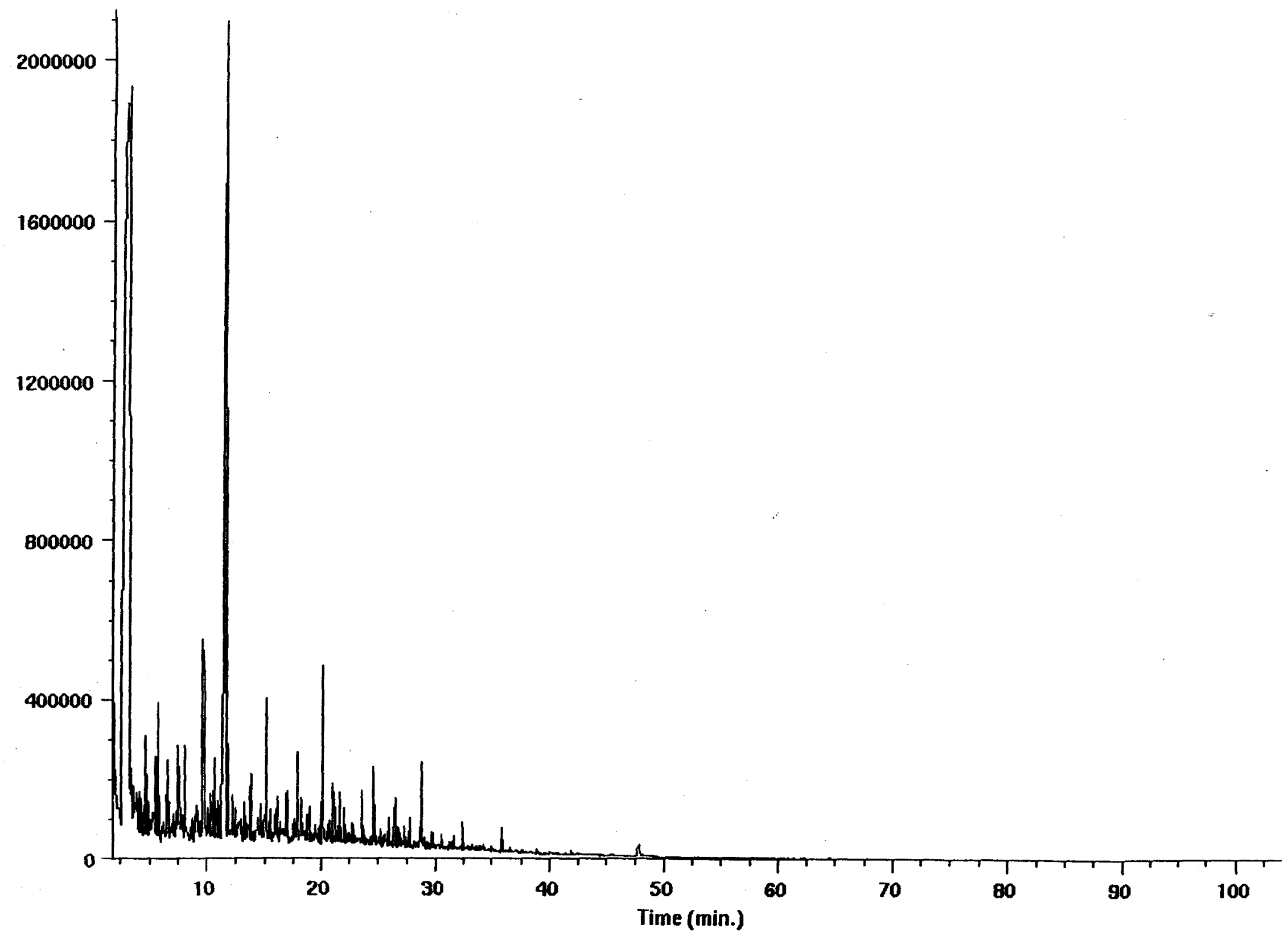




\begin{tabular}{|c|c|c|c|c|c|c|c|c|c|c|c|c|}
\hline Chemical & $\begin{array}{c}\text { Pertention } \\
\text { Tivme }\end{array}$ & & Carcino & genic & ity & $\begin{array}{c}\text { AMES } \\
\text { Mutagenidty }\end{array}$ & Rat Oral & Irritatio & Model & $\begin{array}{l}\text { Developmental } \\
\text { Toxictty }\end{array}$ & Chronic LOAEL & Amount ng \\
\hline Compound & $(\min )$ & $\begin{array}{l}\text { Formab } \\
\text { Mouse }\end{array}$ & $\begin{array}{c}\text { Formale } \\
\text { Rut }\end{array}$ & $\begin{array}{l}\text { Moune } \\
\text { Mouse }\end{array}$ & $\begin{array}{l}\text { Male } \\
\text { Rat }\end{array}$ & & LD 50 & Eye & Stim & & & \\
\hline Tolutene & 4.6 & HGH & No & No & Low & MODERATE & $513 \mathrm{mgkg}$ & SEVERE & MODERATE & NO & $65 \mathrm{mg} / \mathrm{kg}$ & 9.2 \\
\hline Benzene, ethyl-Scaied & 7 & INO & NO & NO & No & MODERATE & $927 \mathrm{mg} / \mathrm{kg}$ & SEVERE & MOOERATE & NO & $68 \mathrm{mg} / \mathrm{kg}$ & 3.5 \\
\hline Benzenc, 1,4-uimethyt-scaled & 7.28 & DNO & NO & $\mathrm{nO}$ & NO & HWH & $925 \mathrm{mg} / \mathrm{kg}$ & SEVERE & SEVERE & 10 & $68 \mathrm{llg} / \mathrm{kg}$ & 8 \\
\hline $1,3,5,7$ cyclooctatetraene- Scaled & 7.9 & $\mathrm{HKGH}$ & NO & NO & HIGH & NO & $45 \mathrm{mg} / \mathrm{kg}$ & MODERATE & MODERATE & NO & $407 \mathrm{mg} / \mathrm{kg}$ & 2.8 \\
\hline benzene, (1-mernyiernyl)-scaled & 8.98 & HGH & No & no & HAGH & mo & $1 \mathrm{~g} / \mathrm{kg}$ & MOOERATE & SEVERE & NO & $218 \mathrm{mg} / \mathrm{kg}$ & 1 \\
\hline Pnenol,4-Metriyt Scaled & 13 & NO & IND & IND & IND & NO & $342 \mathrm{mg} / \mathrm{kg}$ & SEVERE & SEVERE & NNO & $30.9 \mathrm{mg} / \mathrm{kg}$ & 4.5 \\
\hline Phenol, 2 -ethyl-Scaled & 17 & NO & MO & NO & NDD & NO & $658 \mathrm{mg} / \mathrm{kg}$ & SEVERE & SEVERE & ND & $144 \mathrm{mg} / \mathrm{kg}$ & 175 \\
\hline Benzofuran, 2,3-dihydro-Scaled & 18.5 & HMGH & IND & NO & MOOERATE & HGH & $1.9 \mathrm{~g} / \mathrm{kg}$ & MODERAIE & MODERATE & NO & $3.1 \mathrm{mg} / \mathrm{kg}$ & 80.3 \\
\hline Z,4,6-Octatnene, 2,6 dimethyt Scaled & 19 & HGH & HEG & $\mathrm{HGH}$ & HEGH & HIG & $7.3 \mathrm{~g} / \mathrm{kg}$ & MODERAIE & MODERATE & $\mathrm{NO}$ & $65 \mathrm{mg} / \mathrm{kg}$ & 45.5 \\
\hline Cyclotiexene, 1 -ethyt- 6 -ethlidene-Scaled & 19.6 & $\mathrm{HGH}$ & MODERATE & HO & NO & NO & $2.6 \mathrm{~g} / \mathrm{kg}$ & SEVERE & MODERATE & NO & $152 \mathrm{mg} / \mathrm{kg}$ & 12.3 \\
\hline 1.4-benzenedial & 20.1 & $\mathrm{HGH}$ & NO & No & IND & No & $3.6 \mathrm{~g} / \mathrm{kg}$ & SEVERE & MOOERATE & 10 & $4 \mathrm{~g} / \mathrm{kg}$ & 2.5 \\
\hline Pyrazine, methyl-4-oxide Scaled & 20.4 & HEG & NO & No & IND & No & $3.6 \mathrm{gkg}$ & SEVERE & MODERATE & No & $4.1 \mathrm{mg} / \mathrm{kg}$ & 58.5 \\
\hline Ethanone, 1-(2-methypheny)-Scaled & 21 & IND & No & No & NO & HND & $19 \mathrm{mg} / \mathrm{kg}$ & SEVERE & MODERATE & No & $124 \mathrm{mg} / \mathrm{kg}$ & 1.13 \\
\hline Benzeneamine, N N3, 5 -trimethyt-Scaled & 217 & $\mathrm{HEGH}$ & NO & No & HISH & HISA & $1 \mathrm{~g} / \mathrm{kg}$ & SEVEAE & MODERATE & No & $2.7 \mathrm{~g} / \mathrm{kg}$ & 1.5 \\
\hline Ailypheno Scaled & 22 & $\mathrm{HGH}$ & NO & Low & No & No & $260 \mathrm{mg} / \mathrm{kg}$ & SEVERF & SEVERE & HGG & $85 \mathrm{mg} / \mathrm{kg}$ & 33.8 \\
\hline
\end{tabular}


TIC of polycarb2_bsb1.d

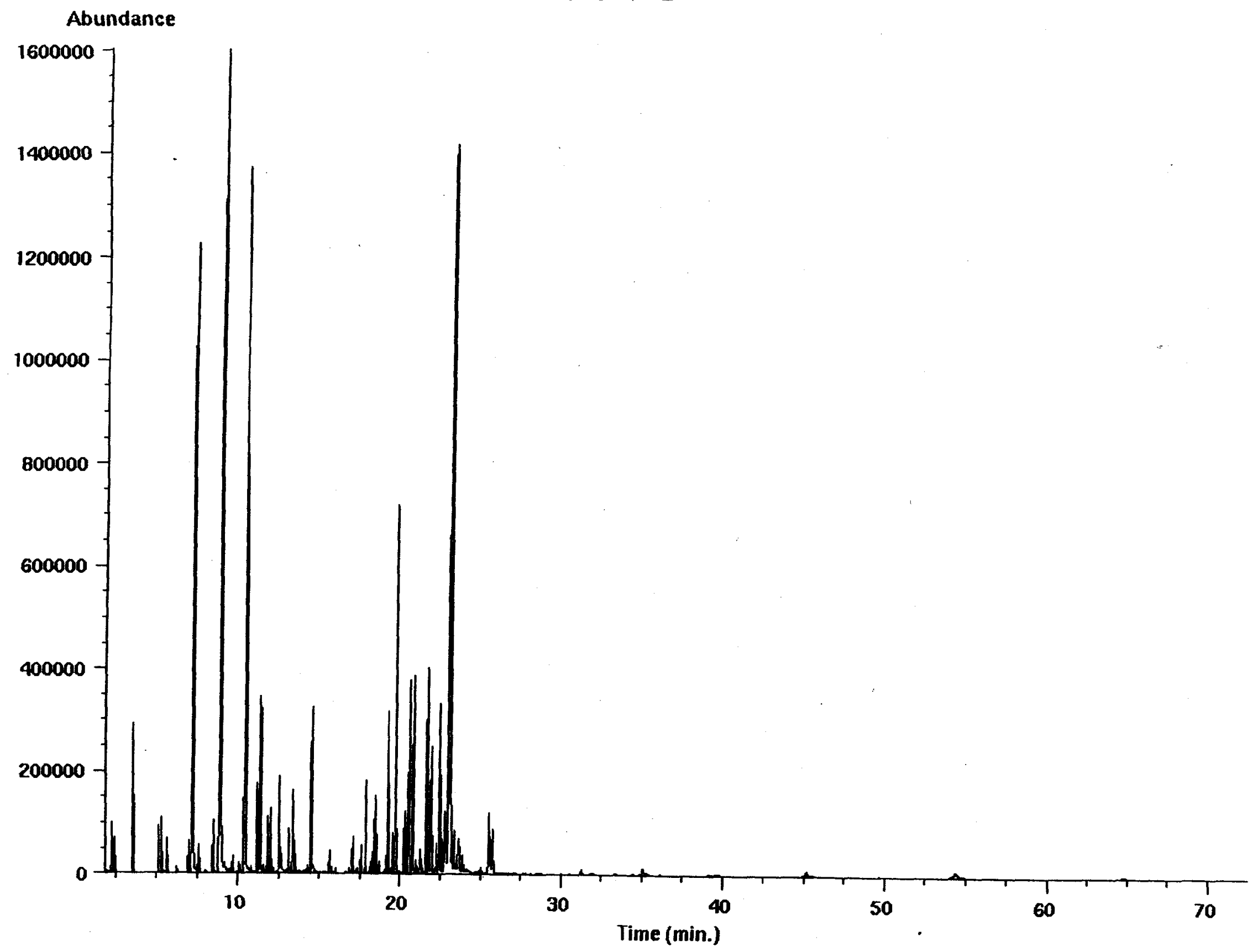




\begin{tabular}{|c|c|c|c|c|c|c|c|c|c|c|c|c|}
\hline Chemical & $\begin{array}{l}\text { Retention } \\
\text { Time }\end{array}$ & & Carcin & genic & & $\begin{array}{c}\text { AMES } \\
\text { Mutagenicity }\end{array}$ & Rat Oral & Irritatio & Model & $\begin{array}{c}\text { Developmental } \\
\text { Toxicity }\end{array}$ & Chronic LOAEL & Amount ng \\
\hline Compound & & $\begin{array}{l}\text { Female } \\
\text { Mounes }\end{array}$ & $\begin{array}{c}\text { Female } \\
\text { Rat }\end{array}$ & $\begin{array}{l}\text { Male } \\
\text { Moune }\end{array}$ & $\begin{array}{c}\text { Male } \\
\text { Rat }\end{array}$ & & LD 50 & Eye & Stin & & & \\
\hline Cyclopentane,metnyi & 2 & Low & HIGH & Low & Low & LOW & $400 \mathrm{mg} / \mathrm{kg}$ & MODERATE & SEVEPE & HGH & & $\cdots$ \\
\hline Toluene & & No & HIGH & Low & Low & NO & $3.3 \mathrm{~g} / \mathrm{kg}$ & MLD & MLD & Low & $18.6 \mathrm{mg} / \mathrm{kg}$ & $-1-\ldots$ \\
\hline Bicyclol 4.2010 cta 135 -triene & & HGG & Low & LOW & $\mathrm{HEH}$ & LOW & $673 \mathrm{mg} / \mathrm{kg}$ & SEVERE & MLD & HIGH & $168 \mathrm{mg} / \mathrm{kg}$ & (n) \\
\hline Benzaldehyde & & HGH & NO & NO & Low & NO & $1.3 \mathrm{~g} / \mathrm{kg}$ & MiL & MID & LOW & $170 \mathrm{mg} / \mathrm{kg}$ & $\ldots$ \\
\hline (1-methylethenyl) benzene & & 10 & No & $\mathrm{HGH}$ & HWGH & No & $3.6 \mathrm{~g} / \mathrm{kg}$ & SEVERE & MLD & LOW & $256 \mathrm{mg} \mathrm{kg}$ & \\
\hline 1,1-(1,3-propanediyl) bis-benzene & & NO & LOW & HGH & Low & NO & $9.80 / \mathrm{kg}$ & MLO & $M \bar{D}$ & $\mathrm{HGH}$ & $68.8 \mathrm{~m} \alpha^{\mathrm{k}} \mathrm{g}$ & $-\cdots$ \\
\hline 6-Phenyt-(E) -2-hexenal & & HGH & LOW & Low & NO & NO & $5.8 \mathrm{q} / \mathrm{kg}$ & MODERATE & MODERATE & HGH & $266 \mathrm{mg} / \mathrm{kg}$ & $\cdots$ \\
\hline 1-methyt2-(2-phenylethenyl)- benzene & & HGH & HGH & Low & Low & NO & $855 \mathrm{mg} / \mathrm{kg}$ & MODERATE & MOOERATE & $\mathrm{HGH}$ & 10 oke & $\ldots$ \\
\hline 1,1-(3-metint-1-propene-1,3-diyl)bibenzere. & & HGH & Low & LOW & MODERATE & Low & $2 g / \mathrm{kg}$ & MODERATE & MODERATE & Low & $10 \mathrm{~g} / \mathrm{g}$ & -1 \\
\hline 1-Benzoyl-4-Piperidione & & LOW & HIGH & Low & Low & NO & $830 \mathrm{mg} / \mathrm{kg}$ & SEVERE & SEVERE & HGH & $197 \mathrm{mg} / \mathrm{kg}$ & $\cdots$ \\
\hline î-methyl-2-phenyl-1h-indole & & NO & HIGH & Low & NO & No & $942 \mathrm{mq} / \mathrm{kg}$ & MODERATE & SEVEPE & LOW & $8 \mathrm{mg} \mathrm{kg}$ & $-\cdots$ \\
\hline $1,1-11-(2,2$-dilnethyt-3-butengl)-1,3-bernene & 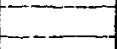 & HGG & MGH & HGH & MOOERATE & HEH & $518 \mathrm{mg} / \mathrm{kg}$ & SEVERE & SEVERE & HGH & $246 \mathrm{mg} / \mathrm{kg}$ & $\ldots \ldots$ \\
\hline Ethanone, 2-hydroxy-1-phebnyl & E. & $\mathrm{NO}$ & No & No & NO & NO & $10 / \mathrm{kg}$ & SEVERE & MODERATE & HEH & $1.3 \% \mathrm{~kg}$ & $\ldots$ \\
\hline 1.1-etrenyidonebis-benzene & 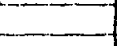 & No & NO & HGH & HIGH & NO & $1.2 \mathrm{~g} / \mathrm{kg}$ & SEVERE & SEVERE & Low & $1.5 \mathrm{mg} \mathrm{kg}$ & - \\
\hline (phenoxymethyy)-benzene & - & No & HGH & LOW & LOW & HIGH & $20 / \mathrm{kg}$ & MOOERATE & SEVERE & HGH & $34.2 \mathrm{mg} / \mathrm{kg}$ & 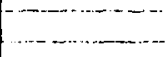 \\
\hline iir-(i-methyl-1,2-ethenediyl) bis-Benzene & & MOOERATE & MGH & HKG & HGH & Low & $1.2 \mathrm{~g} / \mathrm{kg}$ & CBE & CBE & Low & $183 \mathrm{mg} / \mathrm{kg}$ & $-\ldots$ \\
\hline 1,1 -(1-butene-1,4-diyl) bis,(z) benzene & & $\mathrm{HIGH}$ & NO & HWG & NO & NO & $2.5 \mathrm{~g} / \mathrm{kg}$ & CBE & MODERATE & $\mathrm{HGH}$ & $10 \mathrm{~g} / \mathrm{kg}$ & $-\infty-1,-1$ \\
\hline Benzophenone & & LOW & NO & HEH & HGH & NO & $2.10 / \mathrm{kg}$ & SEVERE & SEVERE & LOW & $22 \mathrm{mg} / \mathrm{kg}$ & $\ldots \ldots$ \\
\hline 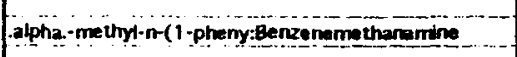 & & HIGH & HIGH & No & Low & HEH & $1.49 \mathrm{~kg}$ & SEVERE & SEVERE & Low & $17.8 \mathrm{mg} / \mathrm{kg}$ & \\
\hline (2-chioro-2-putenyl)- Benzene & -1 & LOW & HUGH & HGH & No & Low & $1.5 \mathrm{gkg}$ & MODERATE & MOQERAIE & HGH & $1.8 \mathrm{~g} / \mathrm{kg}$ & $(-\ldots$. \\
\hline$[1,1$-Biphenyl]-4-carboxaldetiyde & & HGH & Low & NO & LOW & HOH & $1.3 \mathrm{~g} \mathrm{~kg}$ & CBE & SEVERE & Low & $524 \mathrm{mg} k \mathrm{~g}$ & $\ldots \ldots$ \\
\hline [i,1-(1,2-ethenediy!) bis-Benzene & $-\cdots$ & Low & Low & No & LOW & Low & $4.2 \mathrm{~g} / \mathrm{kg}$ & CBE & SEVERE & HGH & $10 \mathrm{~g} \mathbf{k g}$ & $(-\ldots)$ \\
\hline 2- Bromo-1,2-diphenyl-Ethanone & & No & No & $\mathrm{HEG}$ & LOW & No & $1.8 \mathrm{~g} / \mathrm{kg}$ & SEVERE & SEVERE & HGH & $65 \mathrm{mg} \mathrm{kg}$ & 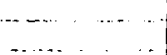 \\
\hline 1-methyt-3-(2-phenyletheny)-(E)-Benzene & & $\mathrm{HIGH}$ & LOW & No & LOW & NO & $4.5 \mathrm{~g} / \mathrm{kg}$ & CBE & MODERATE & $M G H$ & $10 \mathrm{~g} / \mathrm{kg}$ & $-\cdots \cdots$ \\
\hline
\end{tabular}




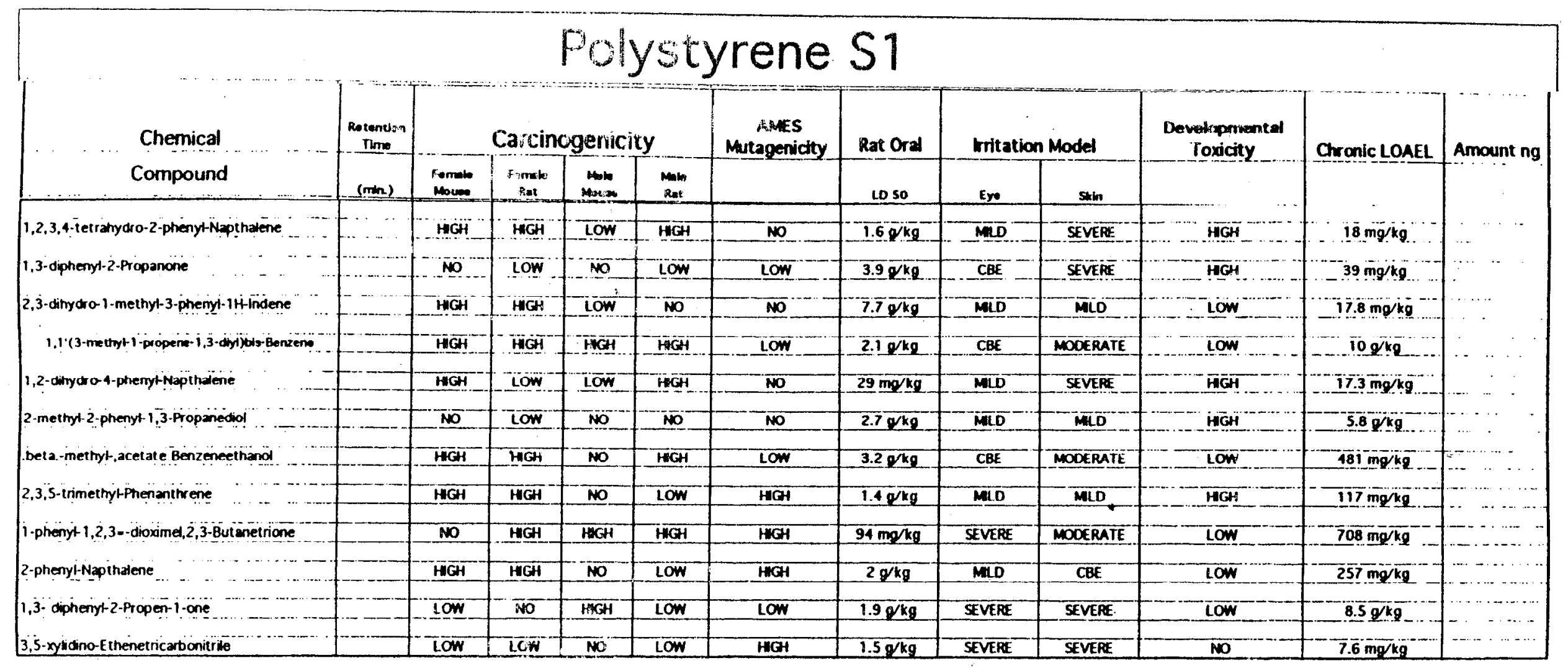


TIC of decurS4.d

Abundance

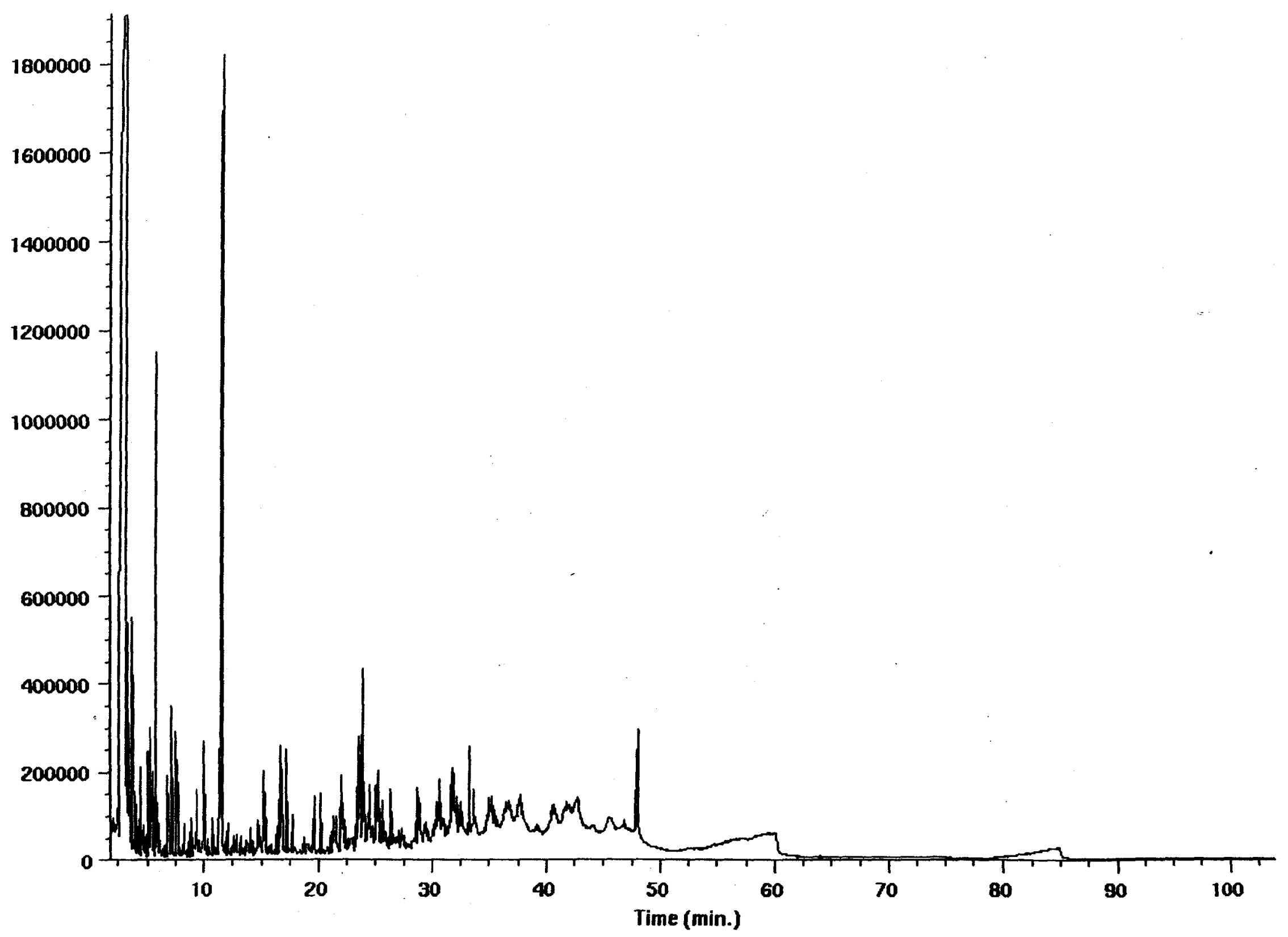




\begin{tabular}{|c|c|c|c|c|c|c|c|c|c|c|c|c|}
\hline Chemical & Rotombion & & Carcino & ggenici & & $\begin{array}{c}\text { AMES } \\
\text { Mutegenicity }\end{array}$ & Rat Oral & Writation & Model & $\begin{array}{c}\text { Developmental } \\
\text { Toxicity }\end{array}$ & Chronic LOAEL & Amount ng \\
\hline Compound & (min.) & $\begin{array}{l}\text { Forratio } \\
\text { Moune }\end{array}$ & $\begin{array}{c}\text { Fomal } \\
\text { Rot }\end{array}$ & Mons & Rut & & LD so & Exe & Sth & & & \\
\hline Ethene,ctioro & 3.2 & $\infty$ & MODERATE & HCH & And & HEG & $537 \mathrm{mo} / \mathrm{kg}$ & $M$ & SEVERE & HWH & $84 \operatorname{mg} k g$ & 56.6 \\
\hline Toluiene & 4.6 & 10 & HEH & DOOERATE & Mo & MO & $3 g / \mathrm{kg}$ & $\mathrm{MD}$ & MD & Mo & $19 \mathrm{mg} / \mathrm{kg}$ & 10.1 \\
\hline Disiloxane, ethenylpentamethyl & 8.6 & HOH & no & No & $\mathrm{HWH}$ & MODERATE & $8.7 \mathrm{~g} / \mathrm{kg}$ & SEVERE & SEVERE & m & $400 \mathrm{mo} / \mathrm{kg}$ & $=-17$ \\
\hline & & & & & & & & & & & & \\
\hline Benzaldehyde & 10.2 & HCH & no & NO & wo & No & $1.3 \mathrm{~g} / \mathrm{kg}$ & MODKRATE & SEVERE & Mo & $170 \mathrm{mg} / \mathrm{kg}$ & 67.6 \\
\hline Phenol & 10.7 & $n$ & No & NO & no & No & $866 \mathrm{mg} / \mathrm{kg}$ & SEVERE & SEVERE & No & $74 \mathrm{~m} g \mathrm{~kg}$ & 30.92 \\
\hline Cyclotet asiloxane, octamethy & 11 & HESH & No & No & HEA & 10 & $70 / \mathrm{kg}$ & SEVERE & SEVERE & HGH & $673 \mathrm{mg} / \mathrm{kg}$ & 175.8 \\
\hline Benzenemethand & 12.53 & no & No & No & no & $\therefore$ & $1.4 \mathrm{~g} / \mathrm{kg}$ & SEVERE & MD & HCH & $556 \mathrm{mo} / \mathrm{kg}$ & 6.8 \\
\hline & & & & & & & & & & & & \\
\hline Cyctotrisiloxane hexamethy & 14 & HEH & No & Mo & no & No & $5.4 \mathrm{~g} / \mathrm{kg}$ & SEVERE & SEVERE & HGH & $550 \mathrm{mq} / \mathrm{kg}$ & 27.7 \\
\hline Hexadecanoic aod 2 pentadeot-1,3-dioxan 5 & 15.6 & MODERATE & No & NO & HWA & $\mathrm{HCH}$ & $3.20 / \mathrm{kg}_{2}$ & MOOGRATE & MOOERAIE & No & $100 / \mathrm{kg}$ & 58.6 \\
\hline Benzeneacetic adid. alpha. 4 -bisil(trimethyls & 75.7 & ND & Mo & No & 10 & mo & $100 / \mathrm{kg}$ & SEVERE & SEVERE & HWH & $126 \mathrm{ma} / \mathrm{ka}$ & 66 \\
\hline & & & & & & & & & & & & \\
\hline Benzoic acid & 17.2 & ND & HOH & 10 & No & 10 & $91 \mathrm{mp} / \mathrm{kg}$ & MOOERATE & MO & $\mathrm{NO}$ & $330 \mathrm{mg} / \mathrm{kg}$ & 2.5 \\
\hline Sidane, $(1,2,3$-benzenetipltrisiony) vis (trim & 19.1 & 10 & 10 & No & MOOERATE & No & $95 \mathrm{mo} / \mathrm{kg}$ & MO & SEVERE & HGH & $126 \mathrm{mg} / \mathrm{kg}$ & 39.6 \\
\hline Cycopentas sioxane, decametry & 22.5 & $\mathrm{HCH}$ & 10 & NO & HGH & No & $9 \% \mathrm{~kg}$ & SEVERE & SEVERE & HOH & $850 \mathrm{mo} / \mathrm{kg}$ & 37.5 \\
\hline Irisitoxane, $1,1,1,5,5,5$ hexameth 13,3 bis & 25.4 & 10 & No & NO & HCH & No & $10 \mathrm{~g} / \mathrm{kg}$ & CBE & SEVERE & HEH & $115 \operatorname{mg} \mathrm{kg}$ & 18.3 \\
\hline 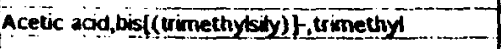 & 27.1 & -10 & No & NO & No & No & $100 / \mathrm{kg}$ & case & SEVERE & No & $285 \mathrm{mg} / \mathrm{kg}$ & $19.1 \cdots$ \\
\hline Einanedicic aod bis(vimeunisiny)ester & 27.4 & HCH & NO & No & No & $\mathrm{FHCH}$ & $3.3 \mathrm{~g} / \mathrm{kg}$ & CBE & MODERATE & no & $409 \mathrm{mg} / \mathrm{kg}$ & 7.97 \\
\hline 4.S-Qhhydobenzo [E] pyene & 32.6 & HIGH & No & no & no & No & $1.7 \mathrm{~g} / \mathrm{kg}$ & SEVERE & SEVERE & No & $23 \mathrm{mg} / \mathrm{kg}$ & $89.2 \ldots$ \\
\hline 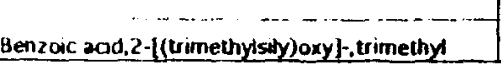 & 37.3 & HHOH. & No & No & $M$ & No & $10 \mathrm{gkg}$ & SEVERE & SEVERE & No & $2 \mathrm{~g} / \mathrm{kg}$ & 23 \\
\hline
\end{tabular}


Abundance

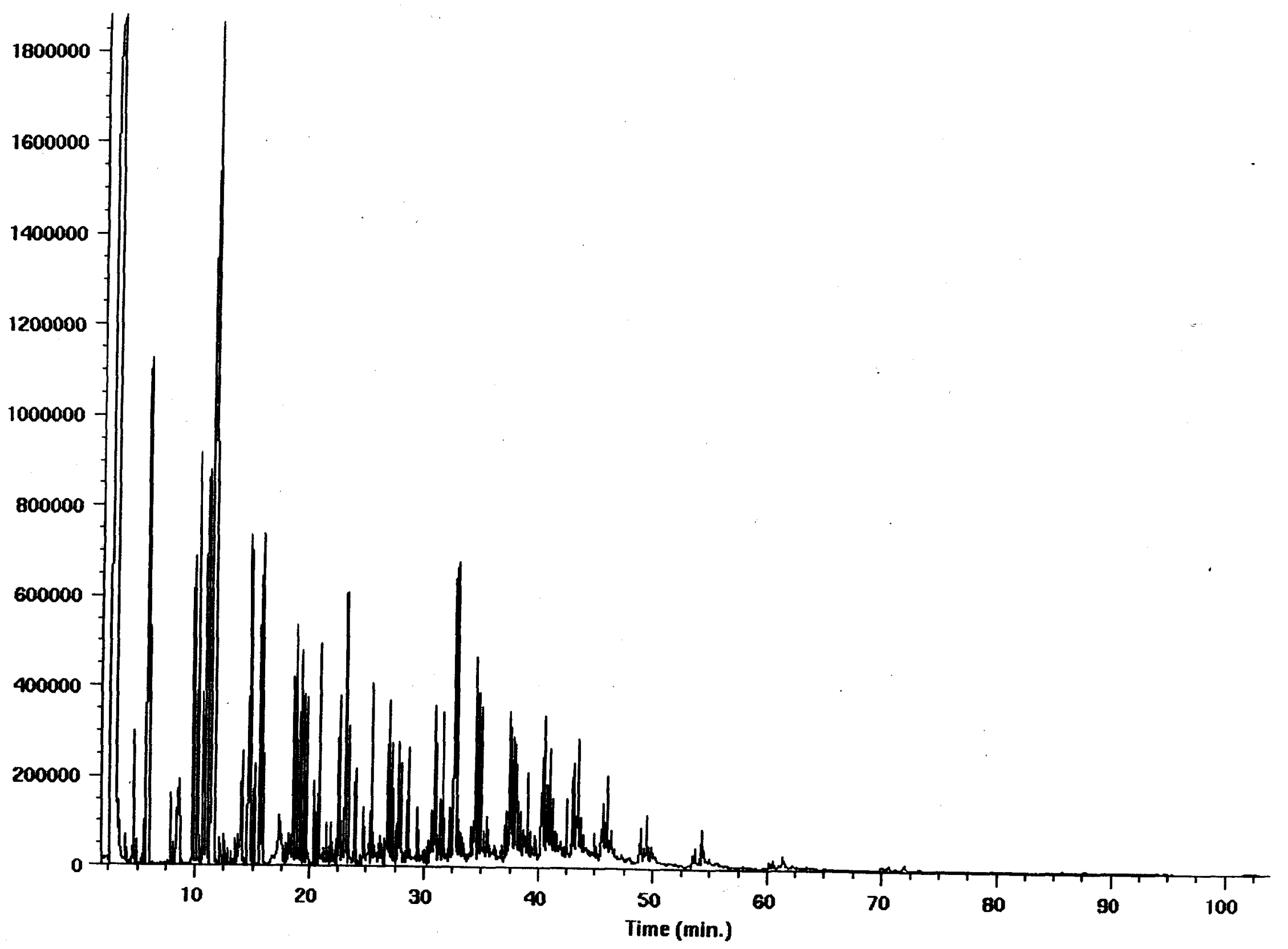




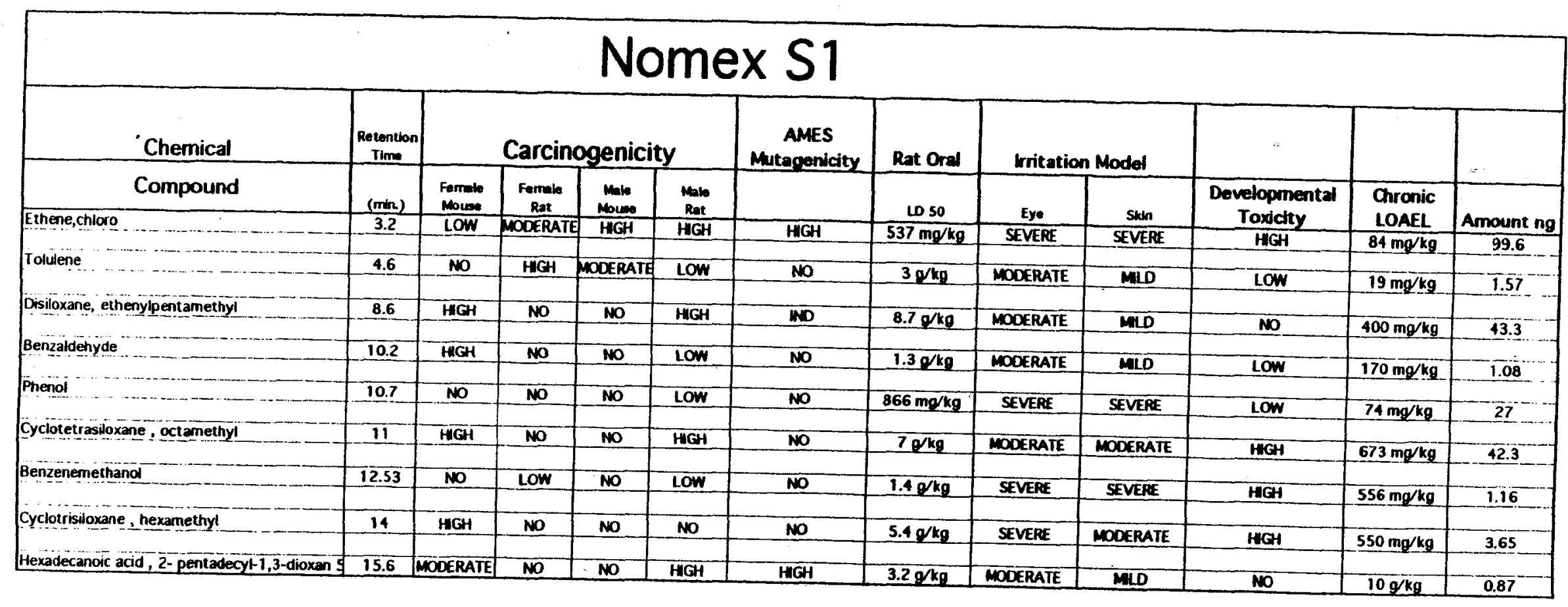


TIC of NomexS1_bsb1.d

\section{Abundance}

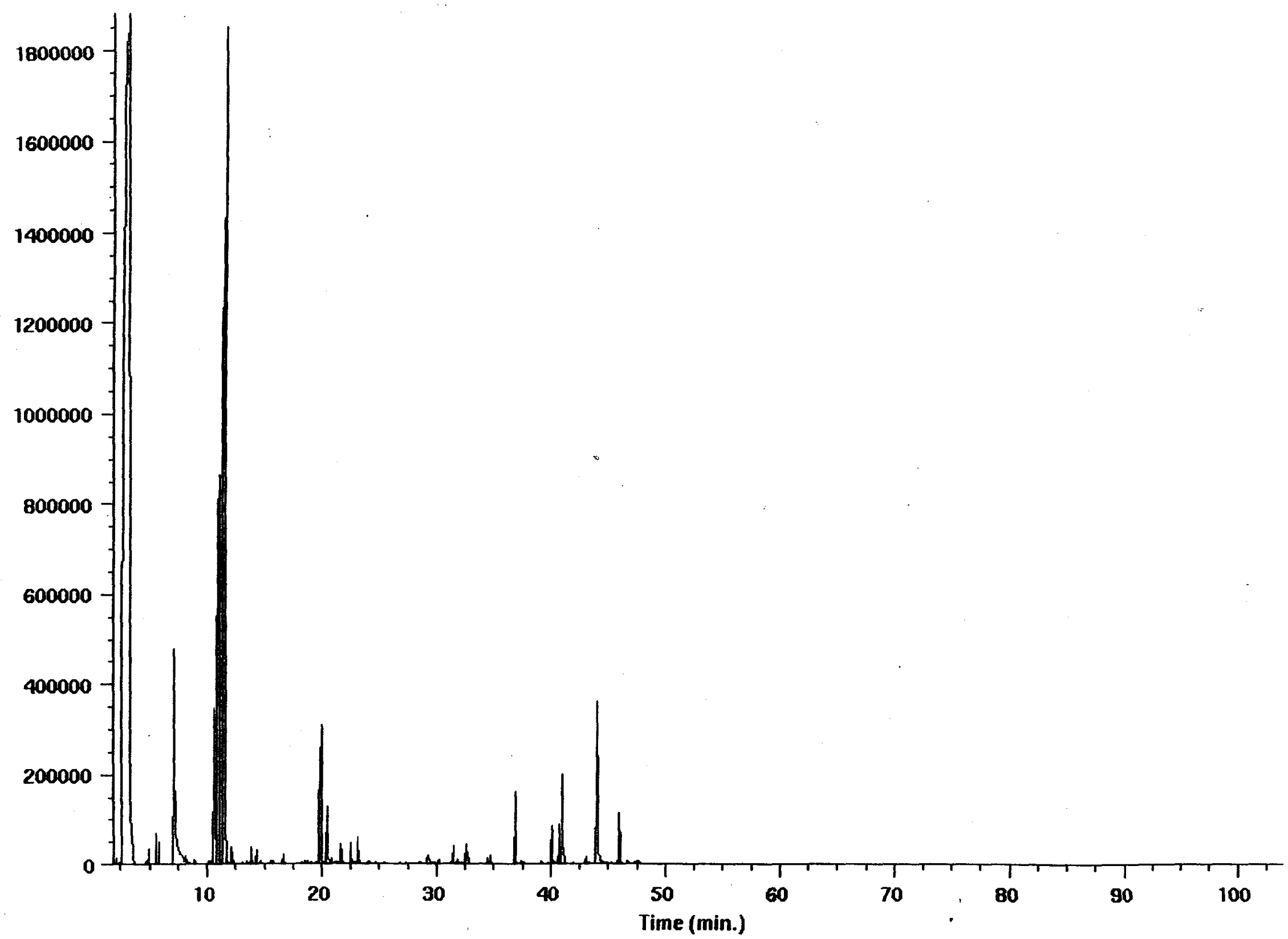




\begin{tabular}{|c|c|c|c|c|c|c|c|c|c|c|c|c|}
\hline Chemical & $\begin{array}{c}\text { Retention } \\
\text { Time }\end{array}$ & \multicolumn{4}{|c|}{ Carcinogenicity } & \multirow{2}{*}{$\begin{array}{c}\text { AMES } \\
\text { Mutagenicity } \\
\end{array}$} & \multirow{2}{*}{$\begin{array}{c}\text { Rat Oral } \\
\text { Lo so }\end{array}$} & \multicolumn{2}{|c|}{ Irritation Model } & \multirow[t]{2}{*}{$\begin{array}{c}\text { Developmental } \\
\text { Toxicity }\end{array}$} & \multirow[t]{2}{*}{ Chronic LOAEL } & \multirow[t]{2}{*}{ Amount ng } \\
\hline Compound & $\frac{(\min )}{30}$ & $\begin{array}{l}\text { Farmalo } \\
\text { Mouse }\end{array}$ & $\begin{array}{c}\text { Fomalo } \\
\text { Rat }\end{array}$ & Mause & $\begin{array}{l}\text { Mole } \\
\text { Rat }\end{array}$ & & & Eye & Stkin & & & \\
\hline 2-Hexand, S-methyt Scaled & 3.8 & IND & NO & NO & HEH & IND & $5 q \mathrm{~kg}$ & SEVERE & MODERATE & HGH & $312.7 \mathrm{mg} / \mathrm{kg}$ & 165.3 \\
\hline Propane,2,2 [ethyyioenebis(oxy)] jois & 5.5 & NO & HGGH & NO & HIGH & WO & $2.7 \mathrm{~g} / \mathrm{kg}$ & MODERATE & No & NO & $351 \mathrm{mg} / \mathrm{kg}$ & 22 \\
\hline 2-Propanone, 1-(1-methylethoxy) & 5.8 & MN & HHGH & No & $\mathrm{HGH}$ & Wo & $3.3 \mathrm{~g} / \mathrm{kg}$ & SEVERE & MD & TNO & simg/kg & 58 \\
\hline Ethanot.2-[2-12-methoxyethoxy]-acetate & 8.2 & HGH & HGH & NO & HGH & NO & $10 \mathrm{~g} / \mathrm{kg}$ & SEVERE & MODERATE & No & $77.3 \mathrm{mg} / \mathrm{kg}$ & 2.5 \\
\hline Oxirane, [(7-methylethoxy)methyl] & 9.6 & IND & HGH & NO & $\mathrm{HHGH}$ & YES & $10 / \mathbf{k g}$ & SEVERE & SEVERE & NO & $354 \mathrm{mg} / \mathrm{kg}$ & 9.6 \\
\hline Azinidine, 1-(methoxymethyl) & 10.7 & ND & HGH & HGH & $\mathrm{HHGH}$ & YES & $1.6 \mathrm{~g} / \mathrm{kg}$ & MLD & MLD & IND & $9 \mathrm{mg} \mathbf{k g}$ & 3.4 \\
\hline Butanoic acid, 4-methoxy-,methyl ester & 12.1 & MD & HGH & No & HIGH & Ino & $8 g / \mathrm{kg}$ & SEVERE & MODERATE & NO & $154 \mathrm{mg} / \mathrm{kg}$ & 5.1 \\
\hline Oxirane, $[(1-$ methylethoxy $)$ methyl $]$ & 12.7 & HD & HGH & NO & HGH & YES & $1 \mathrm{~g} / \mathrm{kg}$ & CBE & CBE & no & $354 \mathrm{mg} / \mathrm{kg}$ & 5.5 \\
\hline 2-Butano, 3,3-oxybis & 12.9 & No & NO & NO & MD & NDO & $4 q \mathrm{~kg}$ & SEVERE & MODERATE & No & $3 \mathrm{~g} / \mathrm{kg}$ & 6.8 \\
\hline 1-Propano, $3-(3-(1-$ methylethoxy $)$ ropoxyl & 14.7 & ino & IND & NO & IND & INO & $6 \mathrm{~g} / \mathrm{kg}$ & SEVERE & MODERATE & NO & $326 \mathrm{mg} / \mathrm{kg}$ & 2.6 \\
\hline Thiocyanic acid, propyl ester & 17.2 & NO & No & NO & No & No & $1.6 \mathrm{~g} / \mathrm{kg}$ & SEVEERE & MODERAIE & HGH & $10 \mathrm{mg} \mathbf{k g}$ & 13.3 \\
\hline 1,3-Dioxare,2-methyl & 20.2 & IND & HEG & HGG & MD & YES & $2 g / \mathrm{kg}$ & SEVERE & MiD & HGH & $258 \mathrm{mg} / \mathrm{kg}$ & 11.5 \\
\hline 2-Propanal, $1-[1$-methyt-2-(2-propenyloxy)ethoxy] 21.1 & & HGH & No & NO & MNO & MO & $9.5 \mathrm{~g} / \mathrm{kg}$ & SEVERE & MODERATE & NO & $261 \mathrm{mg} / \mathrm{kg}$ & 16 \\
\hline Butanoic acid, 3-hydroxy-3-methy & 22 & №. & IND & IND & WD & No & $2.39 / \mathrm{kg}$ & SEVERE & SEVERE & HGH & $300 \mathrm{mg} \mathrm{kg}$ & 4.7 \\
\hline Ethane, 1,2-diethoxy-Scaled & 23.8 & WO & INO & IND & MD & An & $2.7 \% / \mathrm{kg}$ & SEVERE & MID & NO & $240 \mathrm{mg} / \mathrm{kg}$ & 2.1 \\
\hline 1,3-Dioxane, 4,4-dimethy & 25.5 & HGH & $H G$ & № & HGH & NO & $2.2 \mathrm{~g} / \mathrm{kg}$ & SEVERE & MD & HEH & $330 \mathrm{mg} / \mathrm{kg}$ & 3.7 \\
\hline & & & & & & & & & & & & \\
\hline Octane,1-(1-methylethoxy & 25.7 & NO & IND & IND & $\mathrm{HHGH}$ & ND & $8.9 \mathrm{~g} / \mathrm{kg}$ & SEVERE & MODERATE & NO & $20 \mathrm{mg} / \mathrm{kg}$ & 4.1 \\
\hline 2-Decanone-3 hydroxy-3 methyl & 27.3 & No & $\mathrm{NO}$ & No & HGH & No & $3.4 q \mathrm{~kg}$ & SEVERE & MOOERATE & No & $727 \mathrm{mg} / \mathrm{kg}$ & 8.7 \\
\hline & & & & & & & & SEVERE & & & & \\
\hline 2 -teptarione, 3-hydroxy-3-methytscaled & 29.2 & No & NO & MO & ND & NO & $850 \mathrm{mg} / \mathrm{kg}$ & SEVERE & MODERATE & $\mathrm{HIGH}$ & $576 \mathrm{mg} / \mathrm{kg}$ & 3.7 \\
\hline 3-Hexano, 5 -methyt Scaled & 29.4 & IND & NO & MO & HIGH & WNO & $5.5 \mathrm{~g} / \mathrm{kg}$ & SEVERE & MODERATE & MD & $8.8 \mathrm{mg} / \mathrm{kg}$ & 2.3 \\
\hline & & & & & & & & & & & & \\
\hline 1-Propanol,3 $[3-(1-$-methylethoxy)propoxy]-Scaled & 29.5 & NO & IND & No & HEGH & MO & $3.8 \mathrm{~g} / \mathrm{kg}$ & MODERATE & No & NO & $50 \mathrm{mg} / \mathrm{kg}$ & 2.8 \\
\hline Hexose,2,3-dideoxy-5,6-0-(1-methylethylidene) & 32.1 & IND & INO & HGH & IND & & $1.5 \mathrm{~g} k \mathrm{~kg}$ & SEVERE & MODERATE & HEG & $819 \mathrm{mg} / \mathrm{kg}$ & 3.4 \\
\hline rexose, $2,3-$ duseoxy-3,6-0-(1-methylethyldene) & 321 & & & & & YES & & & & & & \\
\hline $2,5,8,11,14,17$-Hexaoxaoctadecane & 33.4 & HIGH & $\mathrm{HHGH}$ & NO & $\mathrm{HHGH}$ & No & $5 \mathrm{~g} / \mathrm{kg}$ & SEVERE & SEVERE & No & $1.4 \mathrm{q} \mathrm{kg}$ & 17.5 \\
\hline 1-Propanal, 3-[3-(methylethoxy)propoxy]-Scaled & 33.8 & IND & INO & IND & HMGH & No & $6 \mathrm{~g} / \mathrm{kg}$ & MOOERATE & MLD & INO & $1.7 \mathrm{~g} / \mathrm{kg}$ & 5.1 \\
\hline
\end{tabular}




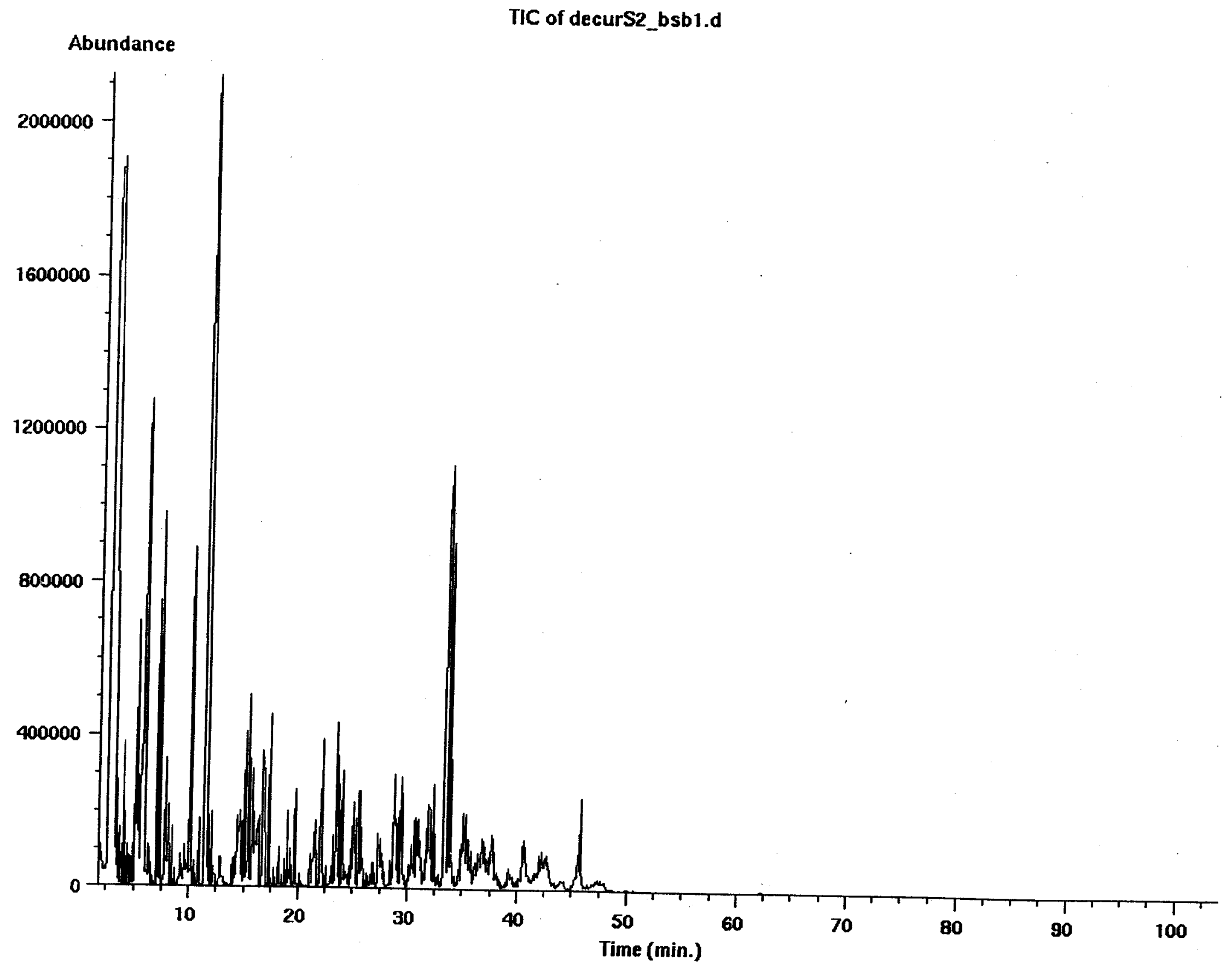




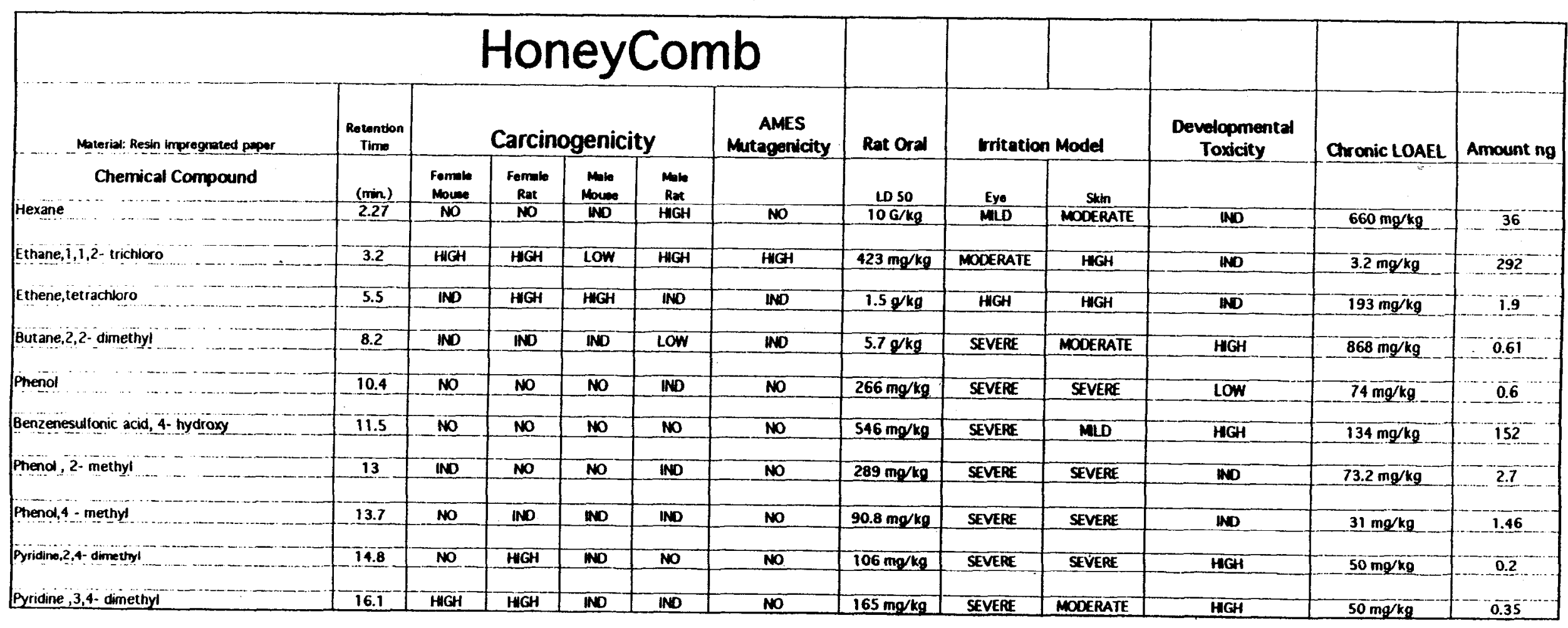


TIC of alq.hocomb.d

Abundance

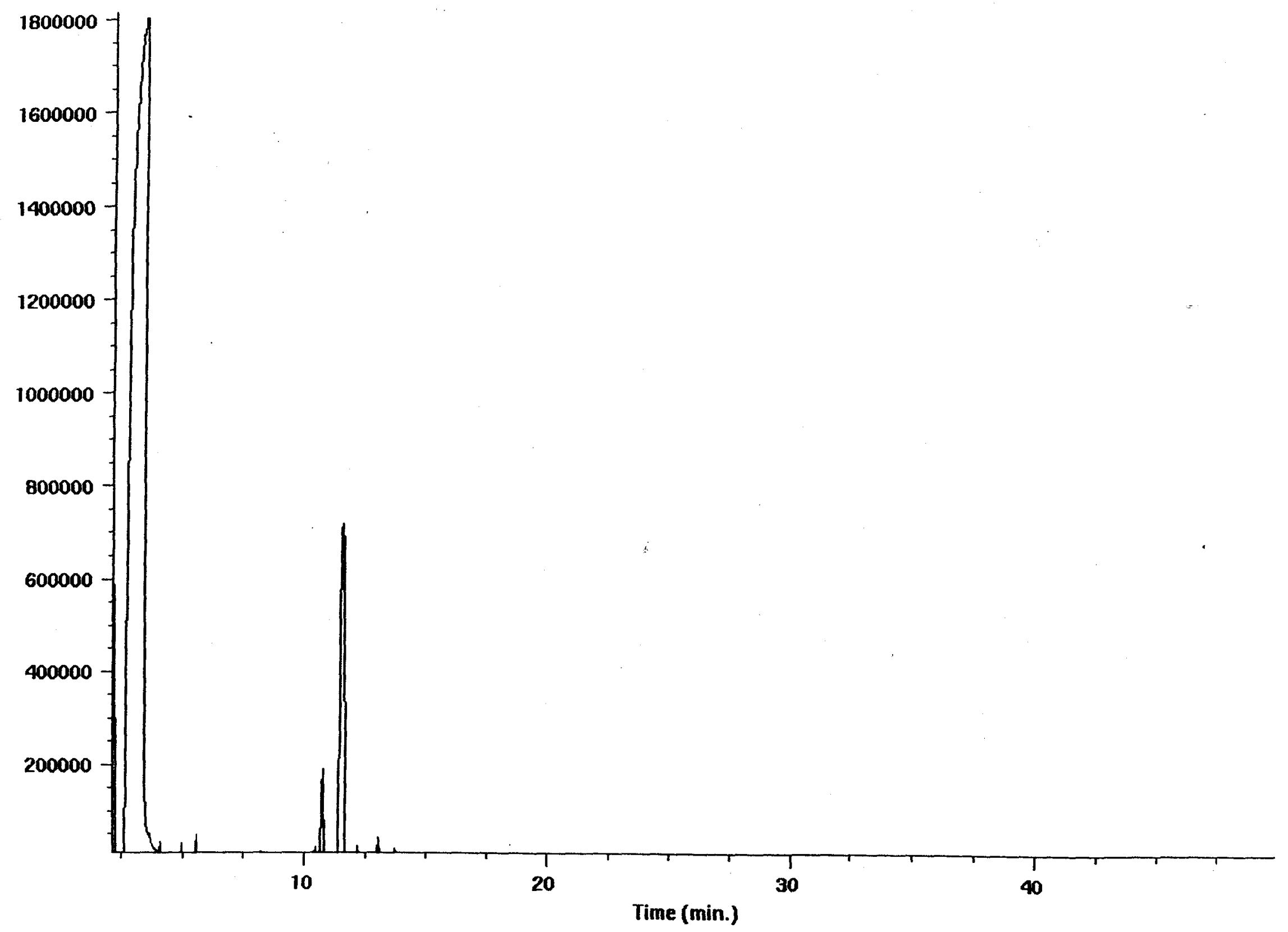




\begin{tabular}{|c|c|c|c|c|c|c|c|c|c|c|c|c|}
\hline \multirow{2}{*}{ 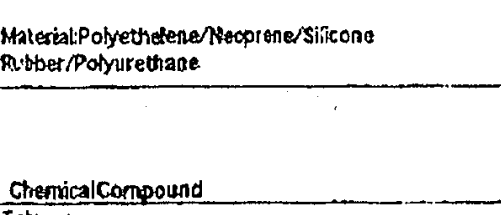 } & \multirow{2}{*}{ 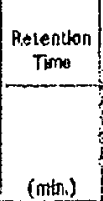 } & \multicolumn{4}{|c|}{ Carcinogenicity } & \multirow{3}{*}{$\begin{array}{c}\text { AMES } \\
\text { Mutagenicity }\end{array}$} & \multirow{2}{*}{$\begin{array}{l}\text { Rat Oral } \\
\text { LD 50 }\end{array}$} & \multicolumn{2}{|c|}{ Irritation Mcidal } & \multirow[t]{2}{*}{$\begin{array}{l}\text { Developmental } \\
\text { Tolidty }\end{array}$} & \multirow[t]{2}{*}{$\begin{array}{l}\text { Chronte } \\
\text { LOAEL }\end{array}$} & \multirow[t]{2}{*}{ Amouning } \\
\hline & & Ferrele & $\begin{array}{c}\text { Fanate } \\
\text { Rat }\end{array}$ & 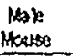 & $\begin{array}{c}\text { Atale } \\
\text { Rat } \\
\end{array}$ & & & & & & & \\
\hline Tolvente & 4.7 & & HaOH & ND & LoN & & $3.3 \mathrm{gkg}$ & MLLO & AHD & L LOWI & $18.6 \mathrm{mg} / \mathrm{kg}$ & 11 \\
\hline Grdoburtanone, 2,3,3-4imetry & 5.2 & Low & LOW & LOW & Lon & NO & $1.7 \mathrm{~g} k \mathrm{~kg}$ & MOO:RATE. & 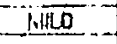 & - & $305 \mathrm{mg} / \mathrm{kg}$ & 26.2 \\
\hline 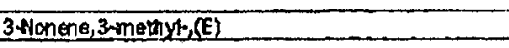 & 5.5 & HGH & LOW & Low & HIGH & 110 & $10 g^{\prime k g}$ & MNOERSARE & Ni? & Low & 229 mokg & 6.4 \\
\hline 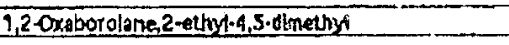 & 5.9 & Low & LOW & $\mathrm{HBOH}$ & $\operatorname{coN}$ & YES & 3,90 & $S$ & SUVRI & II! & $395 \mathrm{mg} / \mathrm{kg}$ & 6 \\
\hline Benzerel, 2-afmethyl & 7.9 & HKGH & MGEH & L.OW & LON & HO & $2.2 \mathrm{~g} k \mathrm{~kg}$ & SEVE & SS VERE & SW & $10.5 \mathrm{mg} / \mathrm{kg}$ & 2.65 \\
\hline 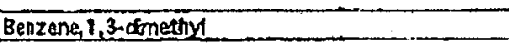 & 8.2 & LOW & HISW & Low & LON & BOO & $2.59 \times 3$ & TVES & SEVER & Low & $6.5 \mathrm{mgkg}$ & 0.76 \\
\hline Benzerieselhyrol & 8.5 & Low & Low & HISH & Low & 10 & $1.5 \mathrm{gkg}$ & RESTME & NEGAM!E & Low & $52 m a / k g$ & 5.3 \\
\hline Ethanestaic sdd, diburty ests & 9 & LON & Low & Low & $1 \mathrm{BOH}$ & No & $2.4 \mathrm{~g} / \mathrm{kg}$ & MEDERATE & $\overline{M O}$ & LOW & $333 \mathrm{mokg}$ & 5.45 \\
\hline & & & & & & & & & & & & \\
\hline $1,3,5,7$-oydooctatraene & 9.3 & HIGH & LOH & Low & $\mathrm{PAOH}$ & NO & 45 mens & AICERATL & MAD & Lr:W & $407 \mathrm{mg} / \mathrm{ma}_{2}$ & 8.7 \\
\hline 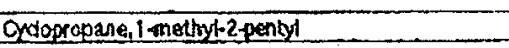 & 9.4 & LON & Loff & Loin & LOW & No & 4 anks. & SFYEPE & MiLO & FED & $484 \mathrm{mg} / \mathrm{kg}$ & 8,75 \\
\hline Butane 1,3 -actloro & 10.1 & HICH I & LOH & HIGH & Low & YES & $2,6 \mathrm{~g} k \mathrm{a}$ & SEVERE & BEVRE & $\mathbb{T N O}^{-}$ & 27 mgkge & 3.25 \\
\hline & & & & & & & & & & & & \\
\hline Propane, 1-chloro & 11.5 & LoW & LON & Loit & Low & YES & $6 g k s g$ & - & MLU & HIGH & 64 mg/xg & 2.3 \\
\hline 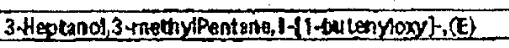 & 12.5 & FEM & Low & LON & $\operatorname{Lon}$ & YES & 10 g/kg & MOESR.TE & SALD & LON & $360 \mathrm{mg} / \mathrm{kg}$ & 7.9 \\
\hline gentzofuran & 74.7 & HoOH & Low & LO'N & LON & YES & $1.5 \mathrm{gik}$ & SEVESE & SEVGRe & Low & $3 \mathrm{mg} / \mathrm{kg}$ & 66.2 \\
\hline 1.Pentanol,2,2,q-aimetry & 15.8 & LOW & LOW & LON & HIGH & 110 & $9.6 \mathrm{~g} k s$ & MLO & $\mathrm{A} Q \mathrm{i}$ & LuN & $30 \mathrm{mg} / \mathrm{kg}$ & $\overline{14.9}$ \\
\hline 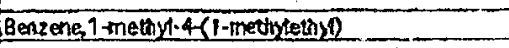 & 76.5 & HIOH & TIOA & LON & HIGH & NO & 2 S 2 . & Win & I!! & Low & $28 \mathrm{mg} / \mathrm{kg}$ & 2.53 \\
\hline 1. Herand, ethyl & 17.2 & WND & Low & LoW & Low & HO & $2 g /: 2$ & IE & SEVERE & . & $371 \mathrm{mg} / \mathrm{kg}$ & 33.6 \\
\hline 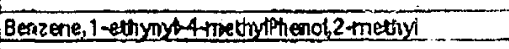 & 17.4 & Low & LOW & HQG & LON & NO & $1.6 \% 3$ & ANUEL $.4 \mathrm{TE}$ & सा10- & Lut & $20 \mathrm{mgkg}$ & 6.8 \\
\hline Phenol, 2 -methyl & 98,4 & Low & Low & LOW & LOW & 10 & $289+10 \%$ & SARE & Mis & - & $73 \mathrm{mg} / \mathrm{kg}$ & 5.3 \\
\hline
\end{tabular}




\begin{tabular}{|c|c|c|c|c|c|c|c|c|c|c|c|c|}
\hline \multirow{2}{*}{ 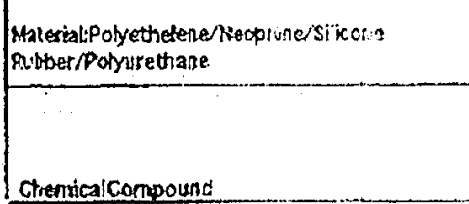 } & \multirow{2}{*}{ 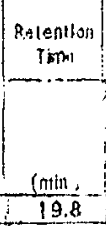 } & \multicolumn{4}{|c|}{ Ceroinogenicity } & \multirow{3}{*}{$\begin{array}{c}\text { AM:S } \\
\text { Mutagenicity } \\
\end{array}$} & \multicolumn{3}{|c|}{ R:Eial | IritatoritMors } & \multirow{3}{*}{$\begin{array}{c}\text { Developmental } \\
\text { Toxicity }\end{array}$} & \multirow{3}{*}{$\begin{array}{l}\begin{array}{l}\text { Chronlc } \\
\text { LOAEL }\end{array} \\
313 \text { mogkg }\end{array}$} & \multirow{3}{*}{ Amount ng } \\
\hline & & 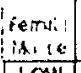 & $\begin{array}{c}\text { Folxals } \\
\text { fit }\end{array}$ & (ka): & Alok & & & $?$ & & & & \\
\hline 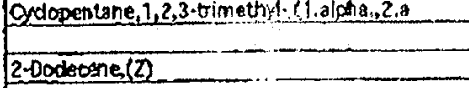 & & Low & LonY & Low & LON & & 3.! & ט.l & MLD & & & \\
\hline 2.Dodeones (2) & $20.7-1$ & HBOA & Low & LOH & Low & YES & -10010 & DUERRT & Uitio & Low & $613 \mathrm{mg} / \mathrm{kg}$ & 3.6 \\
\hline Benzoturala 2- metrol & 21.3 & HaO & LoOS & Low & LON & Yes & $74 \mathrm{mg} \mathrm{kg}$ & SEVIUE & $\$ 118$ & LOW & 14 mogk & 0.92 \\
\hline 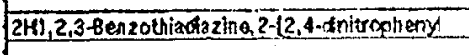 & 22.5 & Loiv & Low & Low & LON & WO & $13=1$ & SET? & MODERATE & Low & 101 magkg & 0.2 \\
\hline 3thexand, 2,2-dimetry & 22.9 & LOW & LOW & LOW & AID & NO & $3.6 x^{2} i t_{2}$ & nornit & $\$$ W1LO & LOW & $923 \mathrm{mg} / \mathrm{kg}$ & 5 \\
\hline fofutand, 4 butcoxy & 23.4 & LOW & LON & LOW & WD & NO & $4 \mathrm{~g} / \mathrm{kg}$ & MOOERATE & $\times 10$ & LOW & $275 \mathrm{mog} / \mathrm{kg}$ & 29.2 \\
\hline 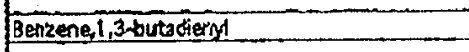 & 24 & HKN & Low & HIGH & LON & YES & $29 / 69$ & XIERATE & BDoED EE & LOW & $57 \mathrm{mo} / \mathrm{kg}$ & 2.9 \\
\hline 4ehthalene, , 2-dinydo & 24,5 & H. WN & HiGH & WN & MDD & No. & $2.4 h g$ & SEYEQR & SEVERE & Low & $42 \mathrm{mg} / \mathrm{kg}$ & 3 \\
\hline Fhend, 3ethrs & 25.1 & LOW & Loir & Low & LOH & No & $3 a / k g$ & SEEEEES & EETPE & Low & $95 \mathrm{mg} / \mathrm{kg}$ & 18.6 \\
\hline Phend,2,2,propessos & 26.3 & LON & LOIY & Low & LON & NES & $-101 \mathrm{~kg}$ & SEVERE & SEVEREA & END & $157 \mathrm{mg} / \mathrm{kg}$ & 4.3 \\
\hline Benzodic ado & 26.8 & L.OW & Hä & LOW & LOW & $\overline{N Q}$ & $39 \mathrm{~kg}$ & MOOORAT & NEGATTVE & LOW & $332 \mathrm{mg} / \mathrm{kg}$ & $\overline{6.62}$ \\
\hline Fhend, $4<(1 \cdot$ methylethy) & 28.9 & HKSH & Low & Low & MDD & Nato- & 371 & MVE & SEVEA & LOW & $116 \mathrm{mag} / \mathrm{kg}$ & 333 \\
\hline Heate thaleng, 2 -mettry & 33.1 & HKOH & HIGH & LOW & LON & YES & $1.4 \pi$ & SisQYe & SNERE: & Low & $67 \mathrm{mg} / \mathrm{kg}$ & 73.6 \\
\hline 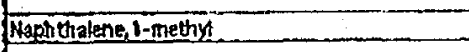 & 32,3 & HKGH & माGH & Low & Lon & YYS & $\mathrm{g} / \mathrm{kg}$ & SEVERE & SERT'PE & LOW & $34 \mathrm{mg} / \mathrm{kg}$ & 9.15 \\
\hline 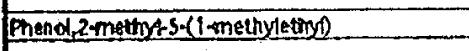 & 32.1 & HKस्I & NDO & Low & IND & No & $4 0 \longdiv { 6 9 }$ & SEVERE- & SEYY:BE & LOW & $102 \mathrm{mg} / \mathrm{kg}$ & 5 \\
\hline 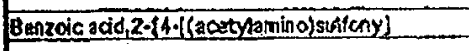 & 33.4 & HGH & Low & HFH & LON & No & $122 \mathrm{mg} n$ & SEVERE & SEISRE & LOW & $6 \mathrm{mg} / \mathrm{kg}$ & $\pi 1.7$ \\
\hline 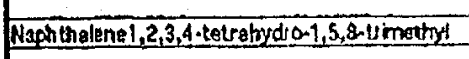 & 36,3 & MाGप्त & LON & LOW & Low & YES & $2.7 \mathrm{~g}^{\prime} \mathrm{kg}$ & SEVERE & इस्पRE & Low & $22.6 \mathrm{mg} / \mathrm{kg}$ & 4.73 \\
\hline 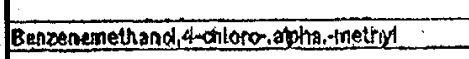 & 37.8 & Low & LON & LO世 & Low & No & $3.69 / . \mathrm{kg}$ & SEVERE & PALL & MOO & 277 mankg & 3.9 \\
\hline 2H-1,2,3-Benzothlodlazzhe,2\}?,4-dnirophen & & & & & & & 83 & M0OOT & 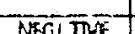 & & $451 \mathrm{~m} / \mathrm{m}$ & \\
\hline (1) & 36,4 & Loir & BifOA-1 & Low & Low & 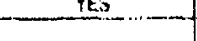 & -3.3968 & DDERALE & WE!! BEE & Hor & $45 f \mathrm{mg} / \mathrm{kg}$ & 9.3 \\
\hline 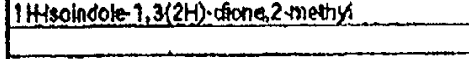 & 39.2 & HOH & LoN & Lat & LOW & $\$ 0$ & $1.2 g^{\prime} \mathrm{kg}$ & WODLRATE & DOOERATE & Low & $14 \mathrm{mg} / \mathrm{kg}$ & 3.25 \\
\hline Naphetialent, Z-ethen & 39.6 & Hiten & HIGH & LOH. & LOW & VES & $3 g^{\prime} 6$ & SEVEES & SEVERE & HूO & $207 \mathrm{ma}^{\prime} \mathrm{kg}$ & 3.12 \\
\hline 1 Nephitho,, 7 , dmettry & 40 & Low & HIGH & LOH & Low & YES & E & SEEVER & SEVERE & LOW & $58 \mathrm{mg} / \mathrm{kg}$ & 1.4 \\
\hline Eliphensterne & 40.5 & HWOH & IND & Low & Low & YES & $130 \mathrm{mg} k$ & -3E & $6 \overline{\mathrm{NE}}$ & LOW & $10 g^{\prime} \mathrm{kg}$ & 5.3 \\
\hline 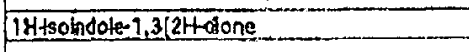 & 42 & HIOH & LoN & - EOW & LOW & Ma & 1017 & STEP & SEver & Low & $19 \mathrm{mg} / \mathrm{kg}$ & 3.36 \\
\hline 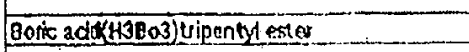 & 42.8 & Low & $10 N$ & Lou & HKGH & wo & ic alke & nownin & 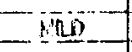 & LOW & 854 makg & \\
\hline & & & & & & & & & & & & \\
\hline
\end{tabular}




\begin{tabular}{|c|c|c|c|c|c|c|c|c|c|c|c|c|}
\hline 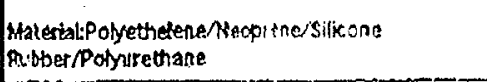 & $\begin{array}{l}\text { Pestentenen } \\
\text { Tinst }\end{array}$ & \multicolumn{4}{|c|}{ Carcimogenicity } & $\begin{array}{l}\text { AMES } \\
\text { Mutagenicit: }\end{array}$ & Rat la! & \multicolumn{2}{|c|}{ Iritation Madel } & $\begin{array}{l}\text { Developmentál } \\
\text { Toxicity }\end{array}$ & $\begin{array}{l}\text { Chronic } \\
\text { LOAEL }\end{array}$ & \multirow[t]{2}{*}{ Amoune ng } \\
\hline Crenticalcompound & (nith.) & perate & $\begin{array}{c}\text { Pantrits } \\
\text { Ris }\end{array}$ & Pats: & $\begin{array}{c}\text { Rake } \\
\text { Ras }\end{array}$ & & 11:5in & Ey: & Skin & & & \\
\hline Phytor & 43.3 & HQGH & HILIS & IIIGH & H164 & $N 0$ & $100 \%$ & MoRAl & NQO & LON & $191 \mathrm{~ms} / \mathrm{kg}$ & -5 \\
\hline gHffucone & 47,3 & HES & $|\mathbb{N}|$ & $10 \%$ & EOH & YES & $67, \cdots 9$ & MEFATE & ACOELATE & LOW & $10 \mathrm{~g} / \mathrm{kg}$ & 3.7 \\
\hline 1 Hexadecente & 48.3 & HGH & LOM & Low & HWG & NO & $10 \mathrm{~g} / \mathrm{kg}$ & !iving & AKQERATE & Loy & $817 \mathrm{mg} / \mathrm{kg}$ & 10 \\
\hline 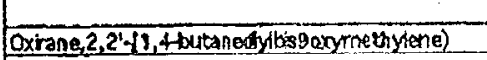 & 49.3 & $\mathrm{HGH}$ & $\mathrm{HIOH}$ & Low & Low & TES & ? $\Omega_{1} x_{1}$ & $-5 y \varepsilon$ & SEMEE & LOW & $10 \mathrm{~g} / \mathrm{kg}$ & 29 \\
\hline Asipholine & $5 a 4$ & $1 \mathrm{HOH}$ & $\operatorname{LON}$ & मानि & HIGH & YYS & 400 ing! i: & SITE & SEVERE & HOSH & $19 \mathrm{mg} \times \mathrm{x}$ & 4 \\
\hline & & & & & & & & & & & & \\
\hline S-atadecene, $\langle E\rangle$ & 52,8 & Lov & Lon & Loy & LON & no & tos & SI:VEPE & MCOSRATE & LOH & $970 \mathrm{mg} / \mathrm{kg}$ & 2.36 \\
\hline Eusan,2.butypetrahycho & 55.8 & LoWf & MMGH & Low & LON & YES & $1 . 3 \longdiv { 6 }$ & Milu & MAS & Low & $598 \mathrm{mg} / \mathrm{kg}$ & 4.4 \\
\hline Antingacene & 56.3 & $\mathrm{EGH}$ & $\mathrm{FH} \mathrm{GH}$ & Low & LON & NO & $2.54 \%$ & SEVERE & MOORATE & HKGH & $234 \mathrm{mg} / \mathrm{kg}$ & 11 \\
\hline Oylododeana, ethyt & 57.5 & Low & LON & Low & Low & NO & $1.7 \mathrm{Ng}^{2}$ & CEVYRE & MHO & LOW & $10 \mathrm{~g} / \mathrm{kg}$ & 2,9 \\
\hline & & & & & & & & & & & & \\
\hline 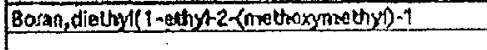 & 61.4 & NOH & HGG & LOHI & $\operatorname{Loth}$ & No & $\operatorname{lng} 2 k$ & SEUERE & $\triangle M O D E R A E$ & Low & $869 \mathrm{mg} / \mathrm{kg}$ & 54.8 \\
\hline Exdohepetan , 1,2-dimethoy trans & 618 & Low & 1.018 & Loll & Low & NO & $59 k 9$ & $-\overline{3 ! 0}$ & MLO & Low & $853 \mathrm{me} / \mathrm{kg}$ & 3 \\
\hline & & & & & & & & & & & & \\
\hline S. HHosene, (E) & 65.6 & Low & Lon & Lon & Loin & No & 108218 & SSVGRE & MDET:ATE: & LOW & $868 \mathrm{mgkg}$ & 6.3 \\
\hline $2,6-0$ tgdene-4, 5 -diol & 88.1 & FRH & LOH & MNO & LaW & YES & Al. $\mathrm{mg} / \mathrm{kg}$ & $-100 \mathrm{RAII}$ & MiLD & LOW & 166 maks & 9.1 \\
\hline Pyrene & 69.7 & Low & Lon & $\operatorname{Lon}$ & CON & No & $31 \times$ & SEVERE & MLO & HGH & $12.4 \mathrm{mgkg}$ & 5 \\
\hline Phenol, 4,4' $4(1$ methytet ry dildene $)$ bs & 73.6 & Low & Low & IND & LON & No & $3 \mathrm{~g}_{\mathrm{kg}}$ & SEYPE & Mill & LOW & $66.5 \mathrm{MG} / \mathrm{KG}$ & 10.5 \\
\hline Esoghthallic soid betyl ester, ester with butol & 74.5 & Low & LOWI & NO & MIOH & NO & $10.4 \sqrt{4}$ & SEYERE & AILD & HKG & $187 \mathrm{mg} / \mathrm{kg}$ & 97 \\
\hline & & & & & & & & & & & & \\
\hline 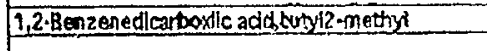 & 79 & Lowi & Lont & Loy & HIOH & 160 & 1069 & EII? & $S H E$ & Low & $389 \mathrm{mg} / \mathrm{kg}$ & 29.5 \\
\hline Methane dis neopentykaxy? & 73.7 & Lov & Lon & Low & MGH & NO & 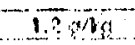 & SUVSE & 5 & LON & $49 ? \mathrm{mg} / \mathrm{kg}$ & 38.3 \\
\hline & & & & & & & & & & & & \\
\hline IEleosene & $B Q 3$ & LOW & Low & $\mathrm{HIGH}$ & DIISH & $\mathrm{NO}$ & $112 \mathrm{k} / \mathrm{ko}$ & $.001+1 \mathbf{E}$ & MLD. & Low & $398 \operatorname{mg} 2 k g$ & 3.15 \\
\hline Trtohenlene & 81.9 & HKGH & LOH & $10 \%$ & LON & YES & $\overline{x+x g}$ & $D^{-1}$ & NEGATVE & $\mathrm{HKH}$ & $11.4 \mathrm{mg} / \mathrm{kg}$ & 7.1 \\
\hline 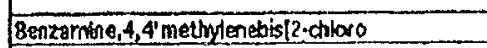 & 82.6 & $\mathrm{HKGH}$ & LOW & HIGH & LON & YES & $1+49$ & I SEYERT & AKHXEATE & Low & $123 \mathrm{mg} \times \mathrm{g}$ & 1.1 \\
\hline 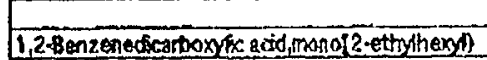 & 82.9 & $\$ 10$ & HIGH & Low & LOW & NO & $2.1 \mathrm{gkg}$ & $18 \mathrm{~J}$ & $M-D$ & Low & $189 \mathrm{mg} \times \mathrm{g}$ & 105 \\
\hline & & & & & & & & & & & & \\
\hline 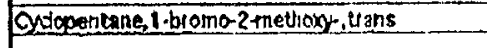 & 84,2 & Low & HiliY! & $\mathrm{HIGH}$ & con & NO & $1: 19 \%$ & SEPE & MAlio & Low & $133 \mathrm{mg} 2 \mathrm{~g}$ & 2.8 \\
\hline 1,2 -Benzenectearboxyfic adot disooctyl ester & 84.5 & LOW & Low & HIGH & MGG & NO & $10 g^{i x}$ & TOE TE & MLLO & HIKH & $9 g / \mathrm{kg}$ & 3 \\
\hline Thensdazde, 4-nitso & 86.7 & HIKH & HGGL & $\mathrm{HIGH}$ & LON & YES & $1.5 \mathrm{~g} / \mathrm{kg}$ & SEVERE & Sever & $\mathrm{HKH}$ & $10 \mathrm{mg} / \mathrm{kg}$ & 155 \\
\hline & & & & & & & & & & & & \\
\hline
\end{tabular}




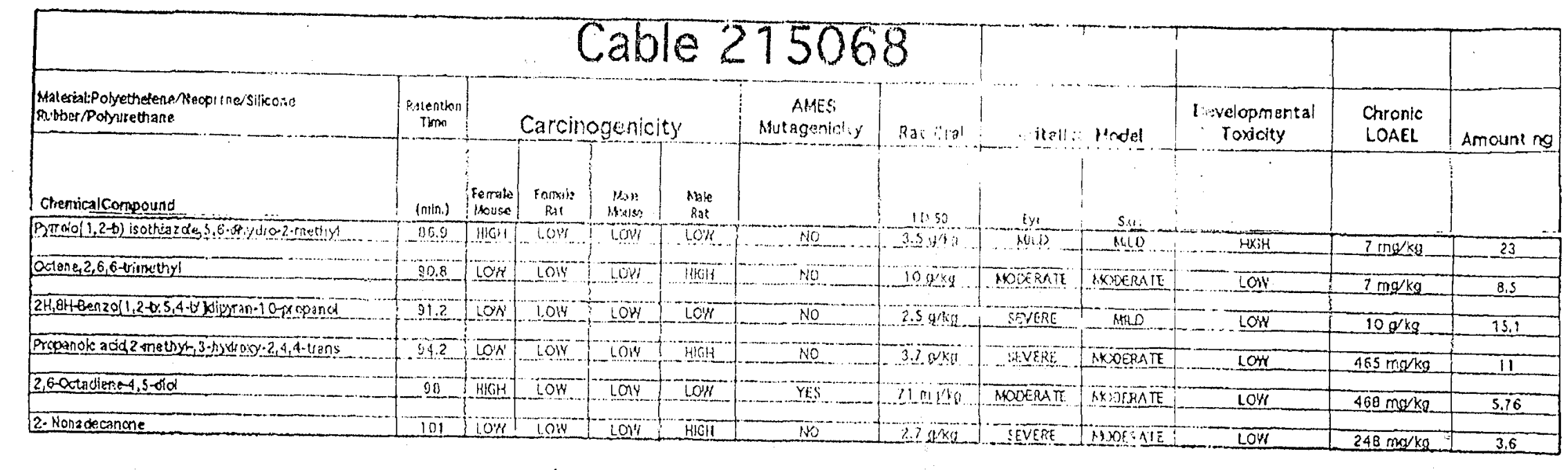




\section{Cable 215258-06}

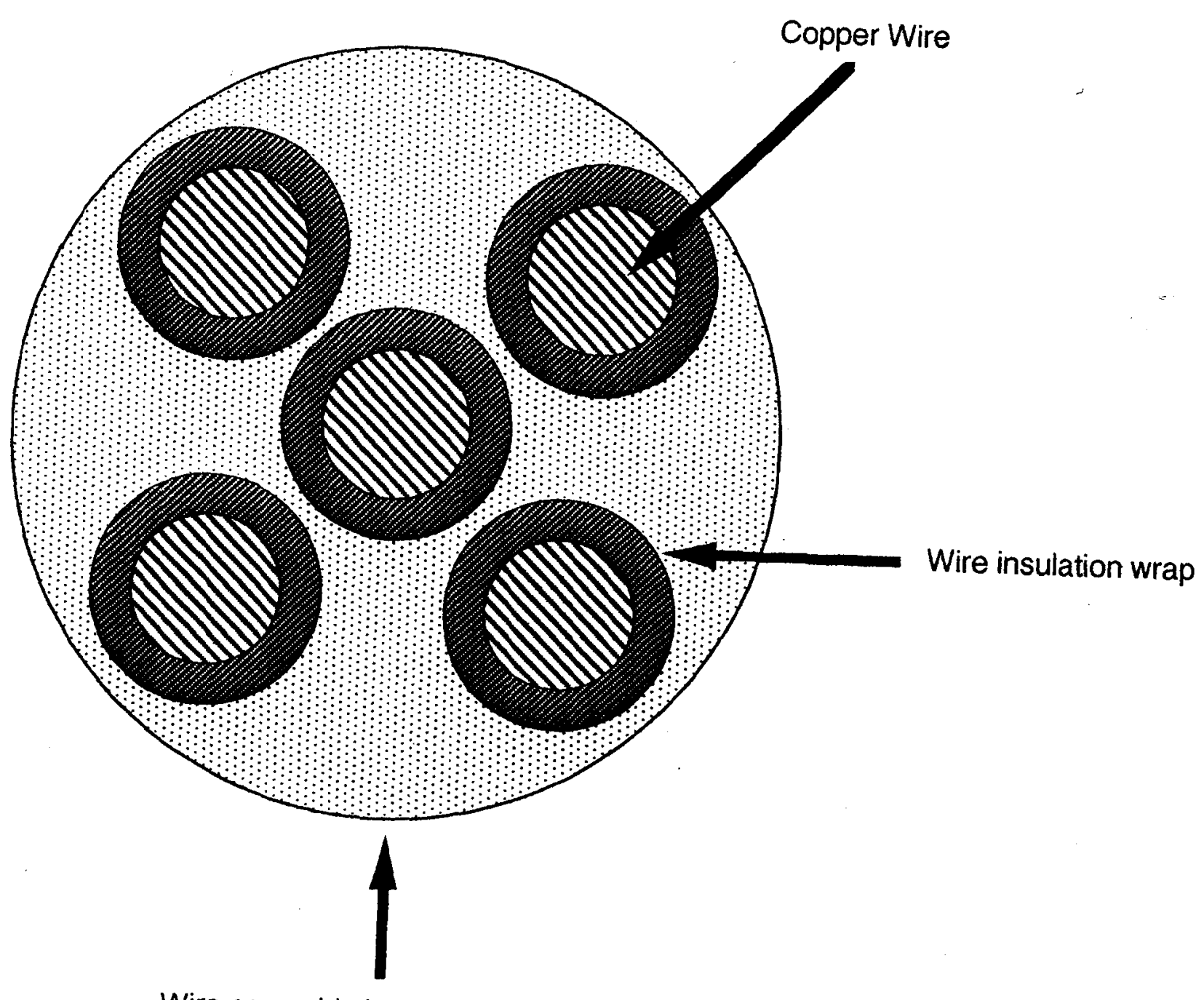

Wire assembly insulation cover 


\begin{tabular}{|c|c|c|c|c|c|c|c|c|c|c|c|c|}
\hline Materiat : nylon,polyurethane, polyvinylidene fluoride & $\begin{array}{c}\text { Retention } \\
\text { Time }\end{array}$ & & Carcin & genic & & $\begin{array}{c}\text { AMES } \\
\text { Mutagenicity }\end{array}$ & Rat Oral & Irritatio & Model & $\begin{array}{c}\text { Developmental } \\
\text { Toxicity }\end{array}$ & $\begin{array}{l}\text { Chronic } \\
\text { LOAEL }\end{array}$ & Amount ng \\
\hline Chemical Compound & (min.) & $\begin{array}{l}\text { Female } \\
\text { Mouse }\end{array}$ & $\begin{array}{c}\text { Female } \\
\text { Rat }\end{array}$ & $\begin{array}{c}\text { Male } \\
\text { Mouse }\end{array}$ & $\begin{array}{l}\text { Male } \\
\text { Rat } \\
\end{array}$ & & $\underline{L} 50$ & Eye & Skin & & & \\
\hline Ethane, 1,2-dichloro & 3.1 & LOW & LOW & $\mathrm{HIGH}$ & $\mathrm{HGH}$ & YES & $2.4 \mathrm{~g} / \mathrm{kg}$ & SEVERE & SEVERE & $\mathrm{HIGH}$ & $84.6 \mathrm{mg} / \mathrm{kg}$ & 97.03 \\
\hline Propane 1,1,2-trichioro & 4.1 & LoW & Low & LOW & LOW & NO & $2.2 \mathrm{~g} / \mathrm{kg}$ & MODERATE & MILD & LOW & $712 \mathrm{mg} / \mathrm{kg}$ & 6.77 \\
\hline Cyclopentanone & $5 . \overline{2}$ & LOW & LOW & LOW & LOW & NO & $1.3 \mathrm{~g} / \mathrm{kg}$ & MODERATE & MODERATE & LOW & $150 \mathrm{mg} / \mathrm{kg}$ & 237.5 \\
\hline Butanoic acid & 8.6 & LOW & HIGH & LOW & LOW & NO & $3.1 \mathrm{~g} / \mathrm{kg}$ & SEVERE & MODERATE & $\mathrm{HIGH}$ & $227.5 \mathrm{mg} / \mathrm{kg}$ & 20.87 \\
\hline 1.4-Butanediol & 9.9 & Low & LOW & LOW & LOW & No & $2.4 \mathrm{~g} / \mathrm{kg}$ & SEVERE & SEVERE & $\mathrm{HIGH}$ & $346 \mathrm{mg} / \mathrm{kg}$ & 23.3 \\
\hline Undecane, 4 4-dimethy! & 22.4 & LOW & LOW & LOW & $\mathrm{HIGH}$ & No & $10 \mathrm{~g} / \mathrm{kg}$ & SEVERE & MODERATE & LOW & $236 \mathrm{mg} / \mathrm{kg}$ & 26.28 \\
\hline $1 \mathrm{H}-1,2,4$-Triazole $=3=$ carboxaldehyde, 5 -methyl & 28.3 & $\mathrm{HIGH}$ & LOW & LOW & LOW & IND & $2.3 \mathrm{~g} / \mathrm{kg}$ & SEVERE & MODERATE & LOW & $206 \mathrm{mg} / \mathrm{kg}$ & 270.68 \\
\hline 2-Cyclopenten-1-one,3amino-2-methyl & 37.9 & LOW & HGGH & LOW & LOW & YES & $2 \mathrm{~g} / \mathrm{kg}$ & SEVERE & MODERATE & $\mathrm{HIGH}$ & $19.8 \mathrm{mg} / \mathrm{kg}$ & 200.6 \\
\hline Fyridine, $2,3,4,5$-tetrahydro & 33.05 & LOW & LOW & LOW & Low & YES & $4.8 \mathrm{~g} / \mathrm{kg}$ & MODERATE & MILD & HIGH & $57.3 \mathrm{mg} / \mathrm{kg}$ & 6.27 \\
\hline Pentanoic acid, 5 -bromo & 34.1 & $\mathrm{HIGH}$ & $\mathrm{HIGH}$ & LOW & LOW & YES & $6.0 \mathrm{~g} / \mathrm{kg}$ & SEVERE & SEVERE & HIGH & $34 \mathrm{mg} / \mathrm{kg}$ & 66.02 \\
\hline 1,6-Dioxacyclododecane-7,12-dion & 38.5 & LOW & LOW & Low & LOW & NO & $3.4 \mathrm{~g} / \mathrm{kg}$ & SEVERE & MODERATE & $\mathrm{HIGH}$ & $958 \mathrm{mg} / \mathrm{kg}$ & 18.77 \\
\hline Propanenitrile, 3 -butoxy & 41.7 & LoW & LOW & LOW & $\mathrm{HIGH}$ & Low & $1.8 \mathrm{~g} / \mathrm{kg}$ & SEVERE & SEVERE & $\mathrm{HIGH}$ & $14 \mathrm{mg} / \mathrm{kg}$ & 10.6 \\
\hline Decanoic acid, silver $(1+)$ salt & 43.6 & Low & LOW & LOW & $\mathrm{HIGH}$ & NO & $8.5 \mathrm{~g} / \mathrm{kg}$ & SEVERE & SEVERE & LOW & $539 \mathrm{mg} / \mathrm{kg}$ & 128.3 \\
\hline 2-0xazolidinone, 3 --ethenyl & 45.3 & $\mathrm{HIGH}$ & $\mathrm{HIGH}$ & HIGH & LOW & YES & $4.7 \mathrm{~g} / \mathrm{kg}$ & SEVERE & SEVERE & $\mathrm{HIGH}$ & $26.3 \mathrm{mg} / \mathrm{kg}$ & 29.3 \\
\hline 1-Propanone, 2 -methyl-1-[2-(1-methylethyl) $c y c 10$ & 74.5 & LOW & LOW & LOW & HIGH & NO & $4.8 \mathrm{~g} / \mathrm{kg}$ & MODERATE & MILD & LOW & $70 \mathrm{mg} / \mathrm{kg}$ & 60.06 \\
\hline
\end{tabular}


Abundance

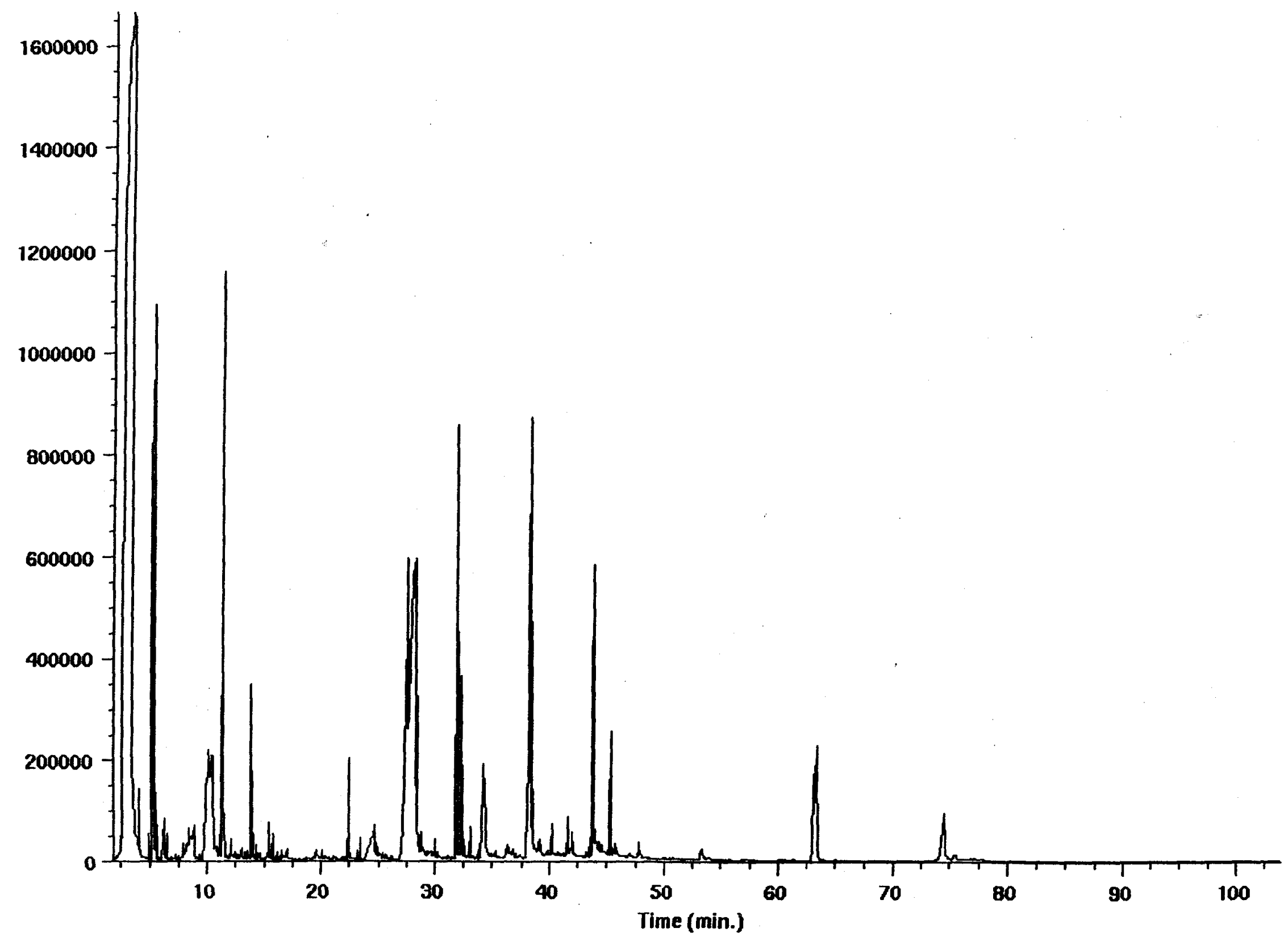




\section{Cable215260-03}

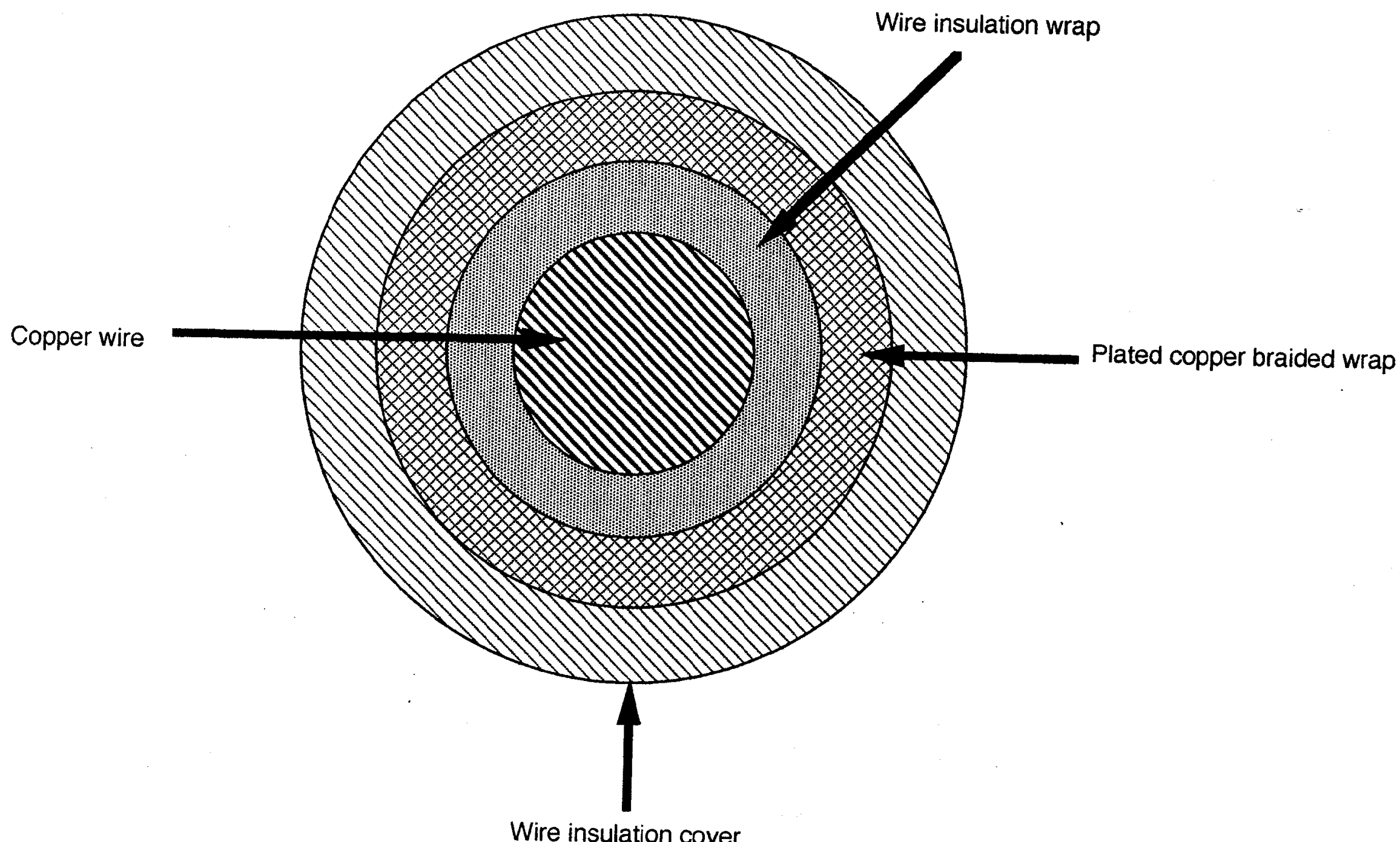




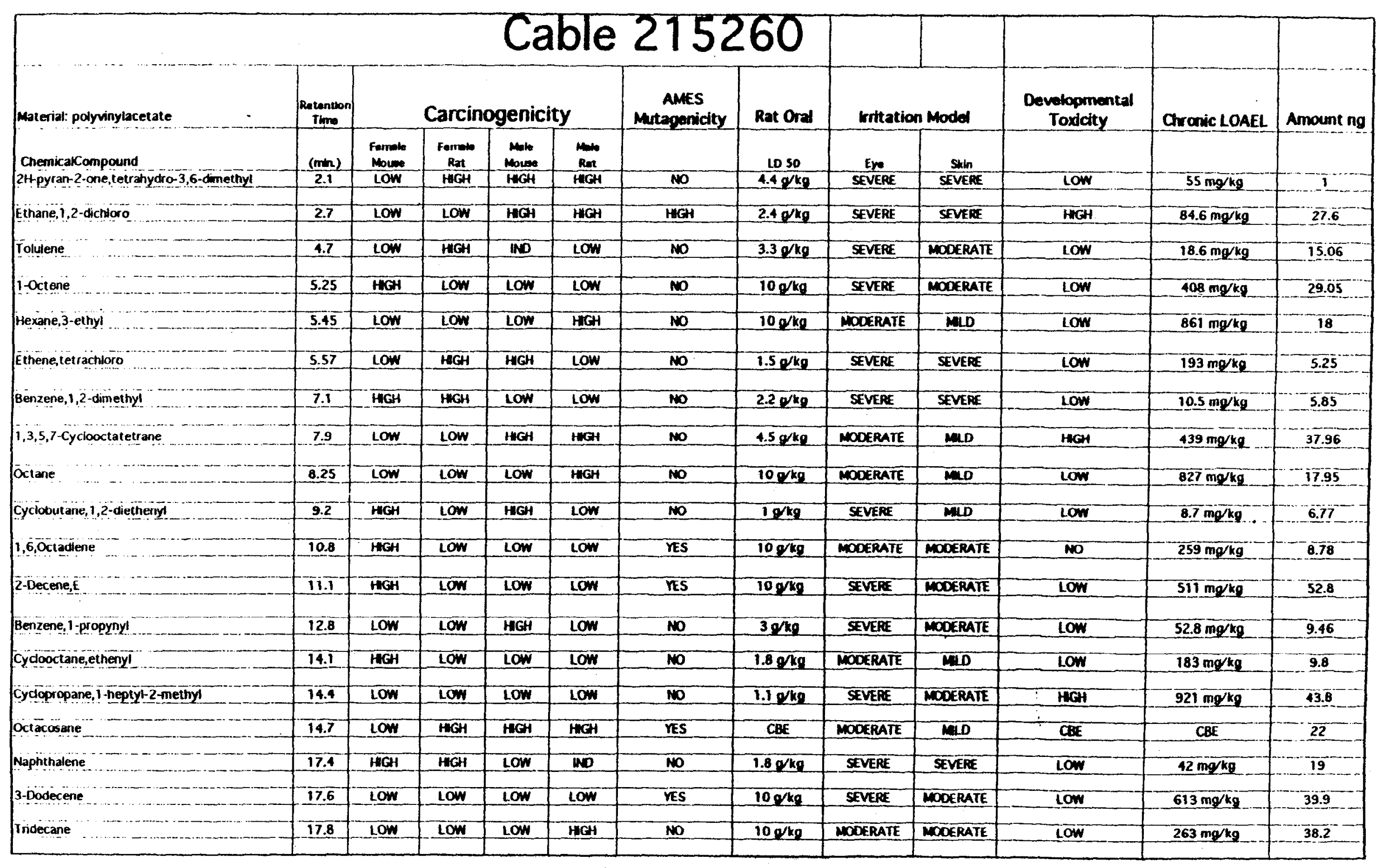




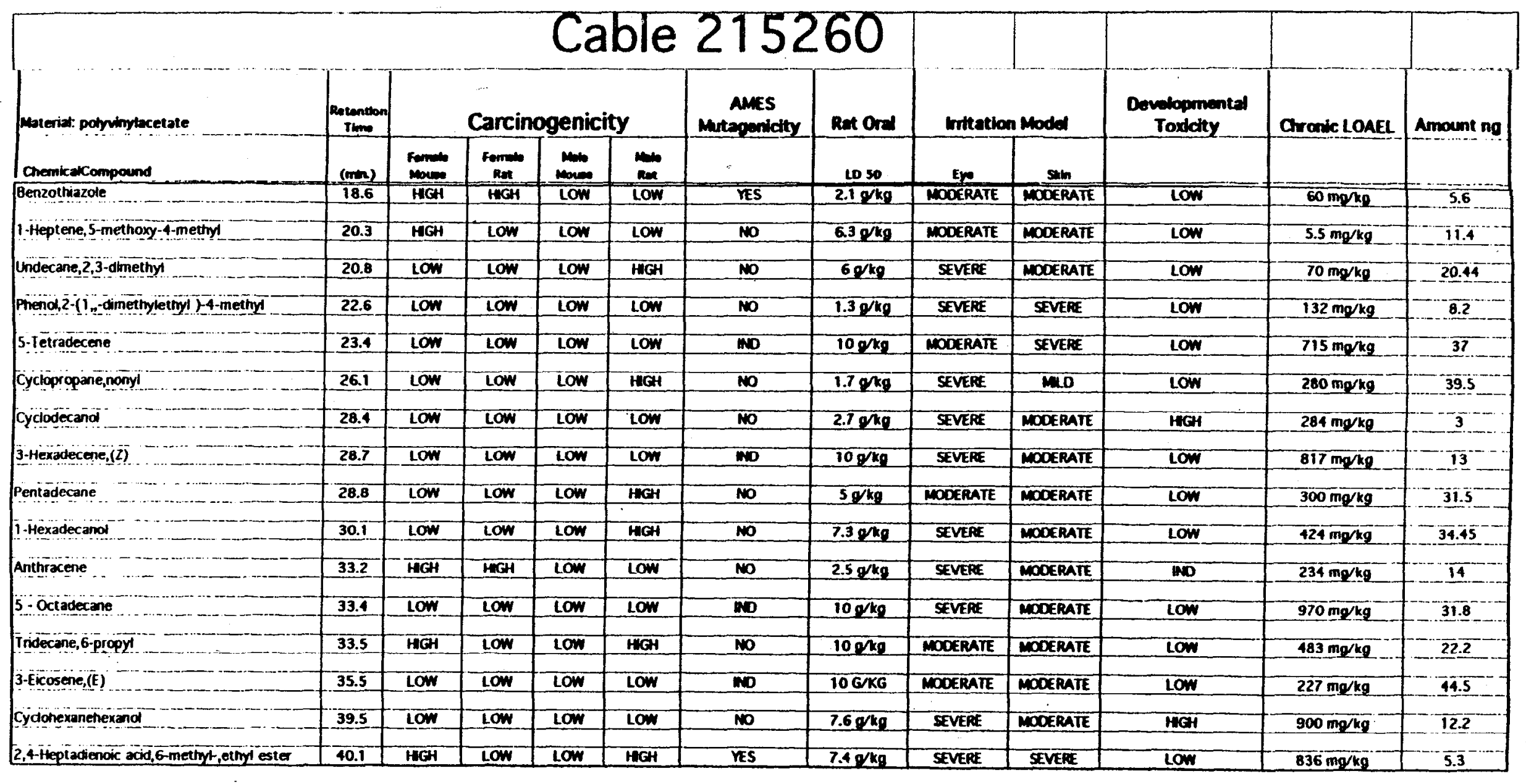


Abundance

TIC of 215260_bsb1.d

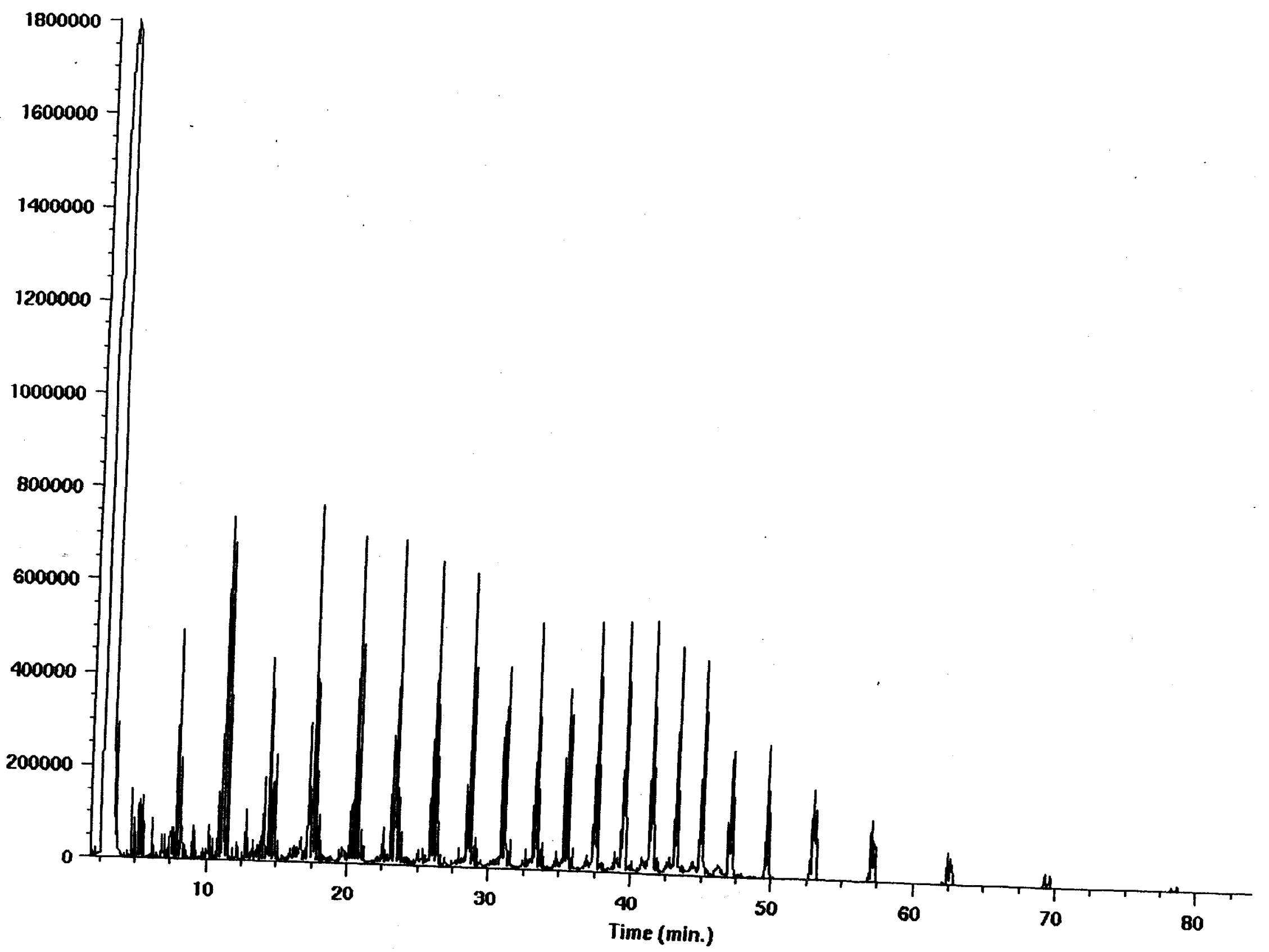




\section{Cable 319161}

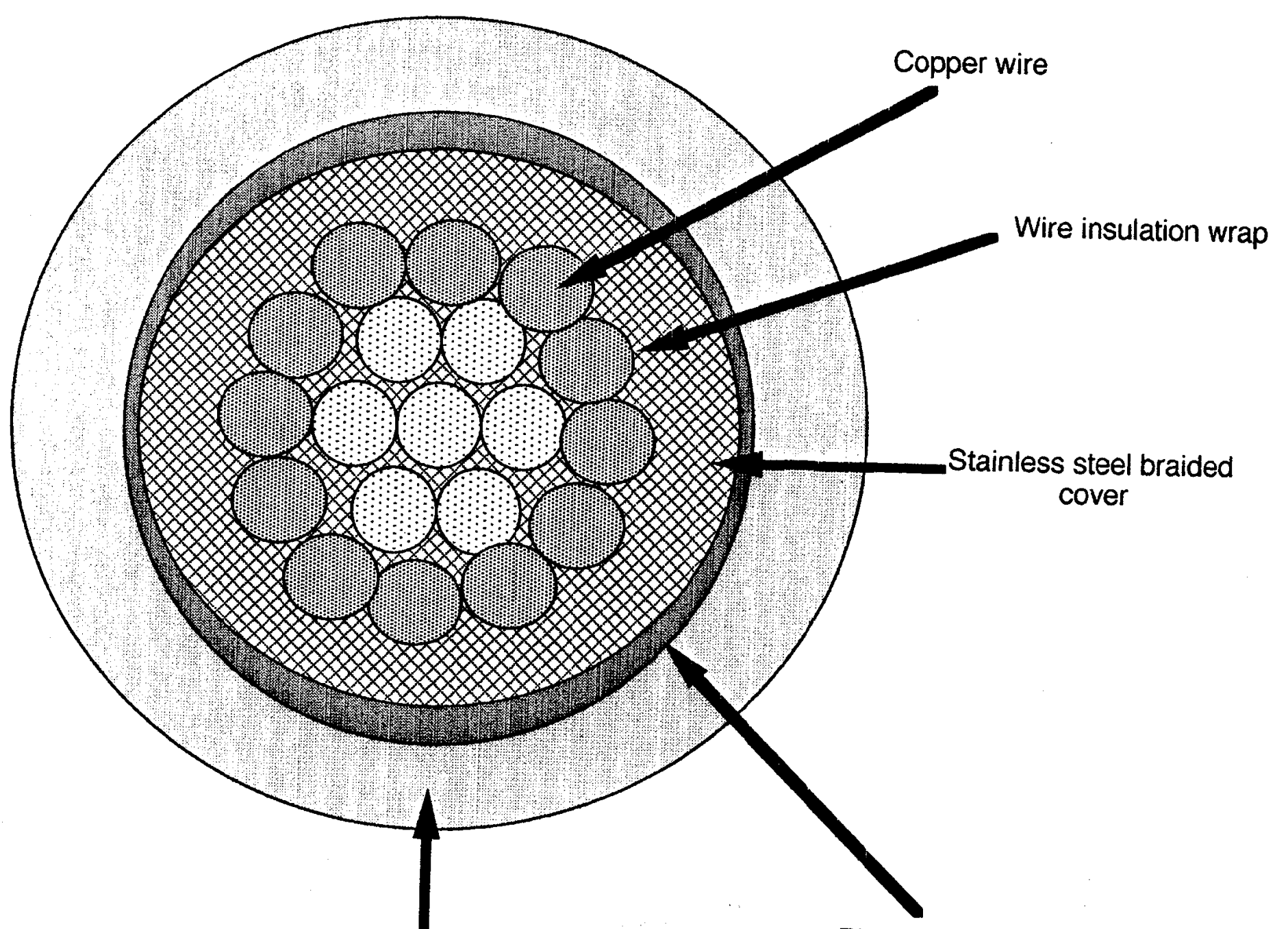

Plastic wrap

Synthetic rubber wire assembly cover 


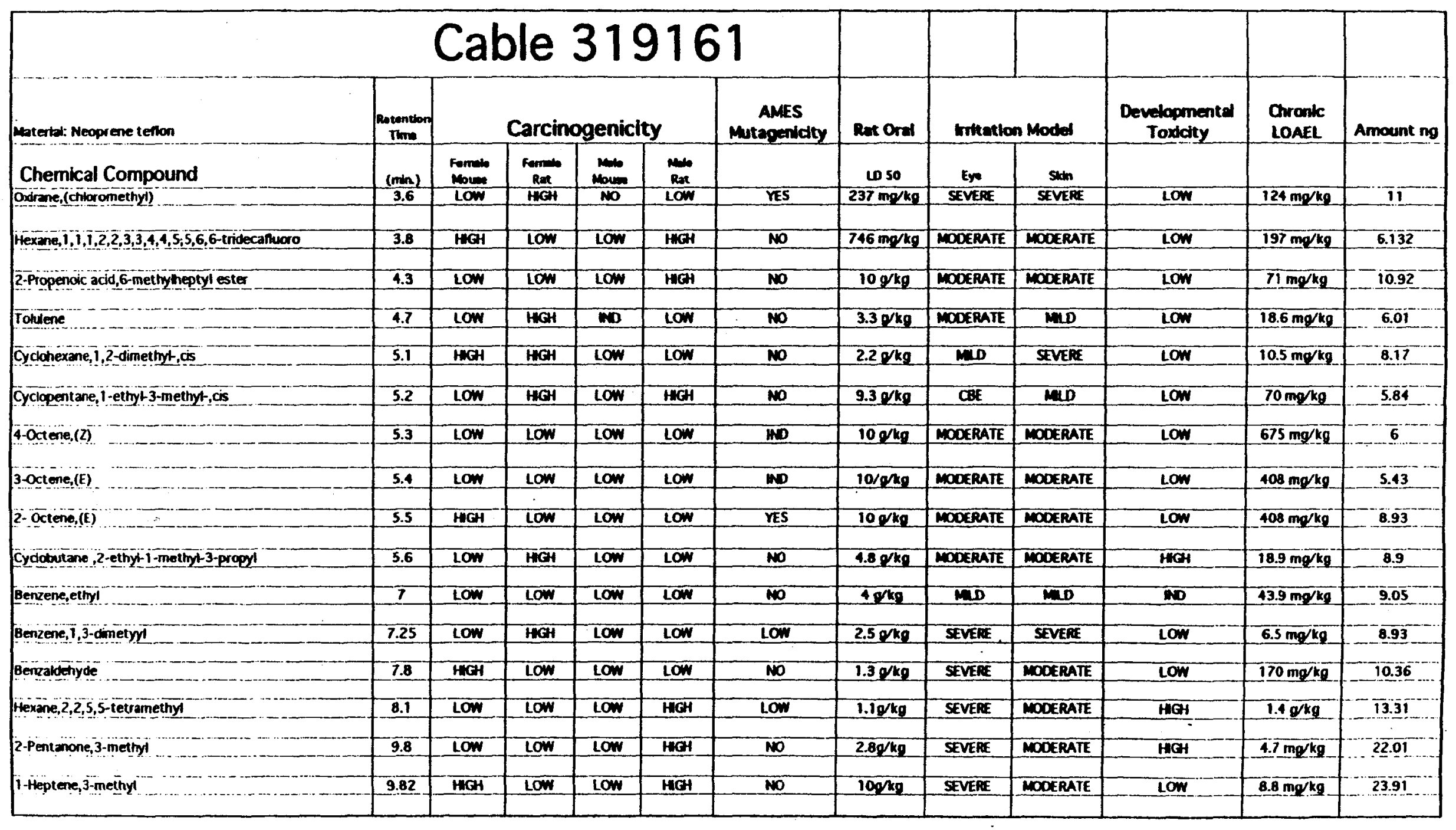




\begin{tabular}{|c|c|c|c|c|c|c|c|c|c|c|c|c|}
\hline Notertat Neoprene tenon & $\begin{array}{l}\text { Rosconton } \\
\text { Tlme } \\
\end{array}$ & & Carcin & genic & & $\begin{array}{c}\text { AMES } \\
\text { Mitagenldty }\end{array}$ & Rat Ore & Ironketio & $n$ Modet & $\begin{array}{l}\text { Developmentel } \\
\text { Toxddry }\end{array}$ & $\begin{array}{l}\text { Cronk } \\
\text { LOAEL }\end{array}$ & Amount ng \\
\hline Chemical Compound & $(\min )$ & forna: & 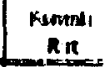 & Moven & $\operatorname{mon}$ & & Loso & ENo & $\sin$ & & & \\
\hline$\ldots \ldots$ & 10.8 & Iow & Low & Low & LOW & 10 & $250 \mathrm{mg} / \mathrm{kg}$ & SEVERE & SEVERE & Low & s!mgkg. & 9.95 \\
\hline Heptene 1,1-oxyot & 12.3 & ow & 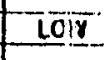 & $\overline{\mathrm{HAH}}$ & HEH & No & $10 \overline{d / 4 x_{0}}$ & ML & $M O$ & Low & $398 \mathrm{mp} / \mathrm{kg}$ & -41.31 \\
\hline onzene (i-melinyt-propeny) $(z)$ & 14 & Lofin & LOW & Low & Low & NO & $3.6 \mathrm{~g} / \mathrm{kg}$ & SEVERE & MOXERATE & $\operatorname{low}$ & $49.6 \mathrm{mo} / \mathrm{hg}$ & 7.85 \\
\hline Benzoon zod & 17.1 & $\tan$ & HOA & Low & Low & No & $3.0 \mathrm{okg}$ & MOOERATE & KEGATIE & Lom & $332 \mathrm{ma} / \mathrm{kg}$ & 64 \\
\hline Naptuatene- & 17.2 & मलA & HWH & Low & no & No & $1.0 \mathrm{~g} / \mathrm{kg}$ & SEVERE & SEVEREE & Low & $42 m q^{20}$ & 10.24 \\
\hline 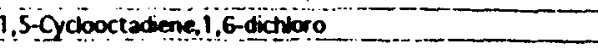 & 18.9 & HOH & INGG & LOW & Low & YES & $5.8 \mathrm{pkg}$ & SEVEEE & SEVERE & Low & $21 \operatorname{mg} k$ & 3.41 \\
\hline Naphthalene, 2-metiny! & 20.6 & Tiiji & HA & LOW & Low & YES & $1.4 \mathrm{gkg}$ & SEVERE & SEVEERE & Low & $67.2 \mathrm{mg} / \mathrm{kg}$ & 6.68 \\
\hline Noprithatene $i$ etimy! - - - - & 23.4 & ar. & Mat & Low & Low & YES & $5, \sqrt[k g]{ }$ & SEVERE & SEVEFe & FWG & $105 \operatorname{mog}$ & -2.36 \\
\hline 1-Butyne, 3-choro - & 23.6 & Hai_ & Low & $\mathrm{HCH}$ & Low & 10 & $2.40 \mathrm{~kg}$ & MOOFRATE & $\mathrm{D}$ & Low & $24.7 \mathrm{mg} \mathrm{x}_{2}$ & 3.82 \\
\hline 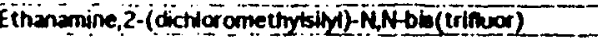 & 28.3 & Low & HGA & HGH & $\mathrm{HCH}$ & 10 & 10 o/ke & SEVEE & SEVERE & HCH & $576 \mathrm{mq} / \mathrm{kg}$ & 183 \\
\hline Hepianentrive & 28.7 & Low & LOW & Low & Low & 10 & 1.3\%/kg & SEVEFE & NGATME & Low & $11.3 \mathrm{mg} / \mathrm{kg}$ & $8.17 \ldots$ \\
\hline 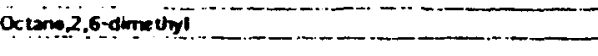 & 39.8 & Fa. & Low & Low & FलH & MO & $100 x_{0}$ & SEVERE & MOOERATE & Low & $60.5 \mathrm{mg} / \mathrm{kg}$ & 3.03 \\
\hline 1,4 Benuenodicutonic add & 35.2 & Low & मित & Low & Low & 10 & $2 \rho / k g$ & SEVEAE & DD & Low & $0 . \overline{0 / k g}$ & 2.3 \\
\hline 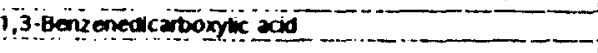 & 35.6 & Int & HGI! & Low & Low & NO & $2.10 / \mathrm{kg}$ & SEVESE & MOOERATE & Low & $\overline{30 i} \mathrm{mg} / \mathrm{kg}$ & 876 \\
\hline Heradocanoic xid & $3 \overline{6.8}$ & EqA & Tun & Low & HGH & 10 & $3.4 \mathrm{pkg}$ & SEVERE & MODERATE & Low & $848 \mathrm{mg} / \mathrm{kg}$ & 9.00 \\
\hline 8 Qunoinol, 2 -meúty! & 37.2 & Lent & HGY & Lon & Low & VES & $20 / k g$ & SEVERE & SEVERE & LOW & $70 \mathrm{mo} / \mathrm{kg}$ & 27 \\
\hline 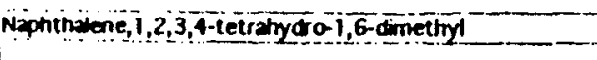 & $3 ? 5$ & Hin & Fi & Low & No & VES & $3.2 \mathrm{~g} / \mathrm{kg}$ & SEVERE. & SEVERE & Law & $26 \mathrm{mg} / \mathrm{kg}$ & 4.55 \\
\hline Benzadotyde 4 nitro onime & 90.7 & A I & wit? & Low & $\mathrm{HOH}$ & NO & $16.50 / \mathrm{kg}$ & SEVERE & STVERE & Low & $31 \mathrm{mg} / \mathrm{kg}$ & $-8.8 i^{-}$ \\
\hline 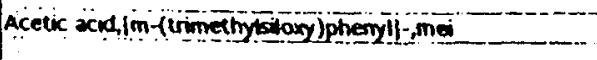 & 41.1 & Sow. & tow: & 1.OW & अन & 10 & 10 oke & MOOERAIE & MD & DAG & $104 \mathrm{mqkg}$ & 31.47 \\
\hline 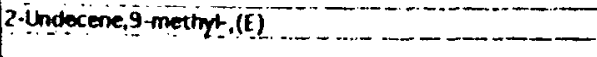 & 8.7 & पm! & $10 \%$ & Low & $10 \mathrm{w}$ & YES & $100 \mathrm{~kg}$ & SAVERE & MOOERATE & ion & $6.2 \mathrm{mpk}$ & $-11+11$ \\
\hline 7-Hexadecyne & 442 & Lovi & tuñ & $10 \mathrm{w}$ & MGH & $\bar{N}$ & 2 & $E$ & MOOERAIE & Low & $90 \overline{0}$ & $14.7 i^{\cdots}$ \\
\hline Oxarame,2,3-bis (1-methylethyl)-urans & 448 & Lat & LOW & Low & HCH & VES & $3.5 \mathrm{~g} \mathrm{~kg}$ & MOOKRAIE & To & MG & $31 \mathrm{mqk}$ & 29.69 \\
\hline Undecane, s-eting-s-propyi & 49.9 & LOW & Logn & on & LOW & $\mathrm{No}$ & $10 \alpha^{k} \mathrm{R}$ & MOOKRAIE & SEVERE & Low & $253 \mathrm{mr} / \mathrm{kg}$ & 3.09 \\
\hline
\end{tabular}


TIC of 319161_bsb1.d

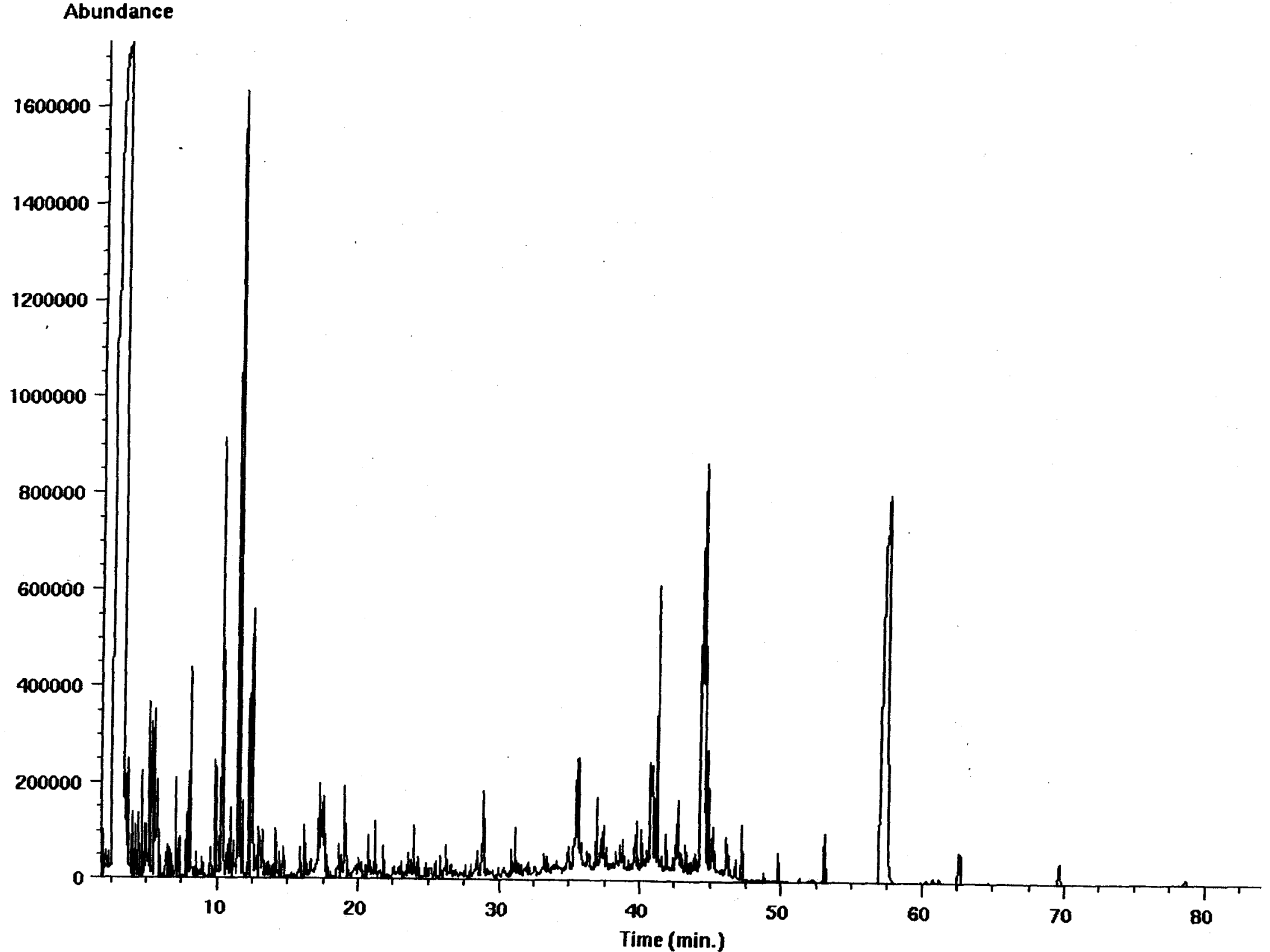




\section{Cable 357566}

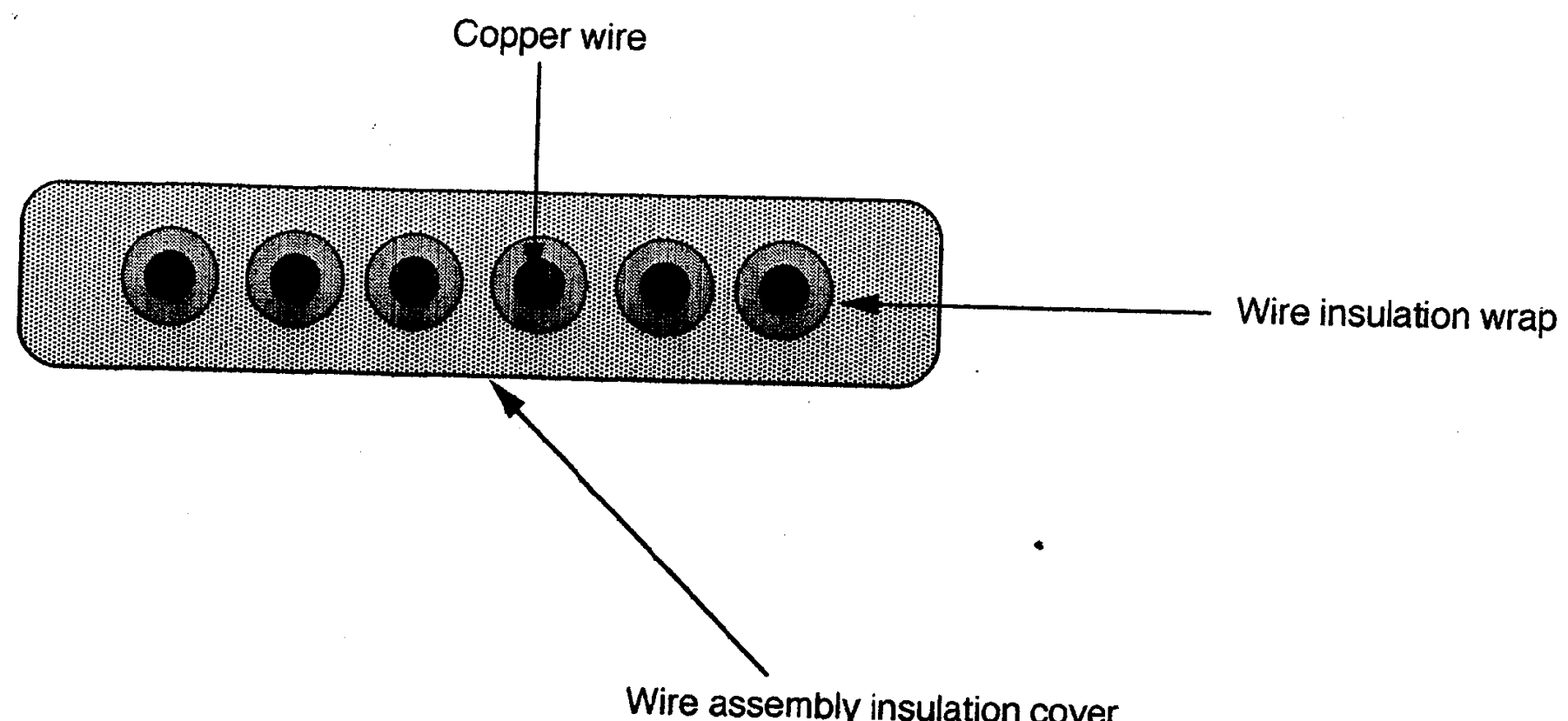

Wire assembly insulation cover 


\begin{tabular}{|c|c|c|c|c|c|c|c|c|c|c|c|c|}
\hline Meterial: teftion polyuretrane & $\begin{array}{c}\text { Retemtion } \\
\text { Thro }\end{array}$ & \multicolumn{4}{|c|}{ Carcinogenicity } & $\begin{array}{c}\text { AMES } \\
\text { Mutagenicity }\end{array}$ & Rat Oral & Iritation & Model & $\begin{array}{l}\text { Dovolopmental } \\
\text { Toxdctity }\end{array}$ & Chronic LOAEL & Amount ing \\
\hline Chemical Compound & & Fomath & $\underset{\text { Rat }}{\text { Romith }}$ & men & $\operatorname{mon}_{\operatorname{Ror}}$ & & & Eye & Stin & & & \\
\hline Ettane, 1,2 - didtoro & 3.1 & Low & MGH & $\mathrm{HCH}$ & $\mathrm{HGH}$ & YES & $119.5 \mathrm{mg} / \mathrm{kg}$ & SEVERE & SEVERE & HGH & $84.6 \mathrm{mg} / \mathrm{kg}$ & \\
\hline Propane $1,1,2$ - trichloro & 4.1 & $\mathrm{HGH}$ & HGH & HGH & LOW & YES & $544 \mathrm{mp} / \mathrm{kg}$ & SEVERE & SEVERE & Low & $87 \mathrm{mg} / \mathrm{kg}$ & \\
\hline Ethene, tetrachioro & 4.8 & Low & IMOH & MGH & Low & YES & $1.5 \mathrm{~g} / \mathrm{kg}$ & SEVERE & MOOERATE & Low & $193 \mathrm{mg} / \mathrm{kg}$ & \\
\hline Propanocic, 2,2-dinethyt,choromethyl ester & 7.4 & Low & $\operatorname{mo}$ & HWG & HGH & YES & $669 \mathrm{mg} / \mathrm{kg}$ & MODERATE & MOOERATE & HGH & $79 \mathrm{mg} / \mathrm{kg}$ & \\
\hline Ether , butyl isopenty & 7.5 & Low & Low & Low & HOH & mo & $7.3 \mathrm{~g} / \mathrm{kg}$ & MODERATE & MOOERATE & Low & $10.6 \mathrm{mg} / \mathrm{kg}$ & \\
\hline Ethenamine, N-nitroso & 8.4 & HGH & Low & Low & $\mathrm{HAH}$ & YES & $52 \mathrm{mg} / \mathrm{kg}$ & MOOERATE & MODERATE & Low & $2.4 \mathrm{mg} / \mathrm{kg}$ & \\
\hline 1,3 Dioxolan - - one, 4,5-dimethyl & 10.4 & Low & Low & FMGH & Low & VES & $1.9 \mathrm{~g} / \mathrm{kg}$ & MOOERATE & MODERATE & HWH & $226 \mathrm{mg} / \mathrm{kg}$ & $\therefore$ \\
\hline Cyclopropane, 1- propenyl & 11.1 & ino & Low & no & ton & No & $2100 \mathrm{~kg}$ & STYPFE & SFYEFF & 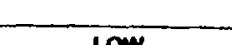 & & \\
\hline & 73 & & & & & & & & & & 139 minke & \\
\hline Furan, 2-butyutrahydo & 13.3 & Low & HGH & Low & 00 & YES & $1.3 \mathrm{~g} / \mathrm{kg}$ & MODERAIE & MOOERATE & LOM & $598 \mathrm{mg} / \mathrm{kg}^{-}$ & \\
\hline Heptane 2,6-dimethyl & 13.6 & HGH & Low & Low & LOW & No & $0.2 \mathrm{gkg}$ & MODERATE & MOOERATE & HGH & $1.2 \mathrm{~g} / \mathrm{kg}$ & \\
\hline 1- But anol, 4-butoxy & 15.7 & Low & Low & Low & no & VES & $3.9 \mathrm{gkg}$ & MODERATE & MOOERATE & Low & $1.3 \mathrm{~g} / \mathrm{kg}$ & \\
\hline 2- Butene, I-propoxy & 16.1 & HGG & Low & Low & LOW & YES & $4.3 \mathrm{~g} / \mathrm{kg}$ & MODERATE & MODERATE & Low & $.64 \mathrm{mg} / \mathrm{kg}$ & \\
\hline Ooriane butyl & 16.3 & Low & HEH & Low & $\overline{M_{0}}$ & YES & $836 \mathrm{mg} / \mathrm{kg}$ & SEVERE & SEVERE & Low & $850 \mathrm{mg} / \overline{\mathrm{kg}}$ & \\
\hline Naphthatene & 17.2 & HGH & HGH & Low & Low & No & $1.8 \mathrm{~g} / \mathrm{kg}$ & SEVERE & MOOERATE & LOW & $42 \mathrm{mg} / \mathrm{kg}$ & \\
\hline Furan , tetrajhydó-2-mothyl & 19.4 & Low & मGH & मCH & No & YES & $765 \mathrm{mo} / \mathrm{kg}$ & MODERATE & DOOERATE & $\mathrm{HCH}$ & $290 \mathrm{mo}$ & \\
\hline
\end{tabular}




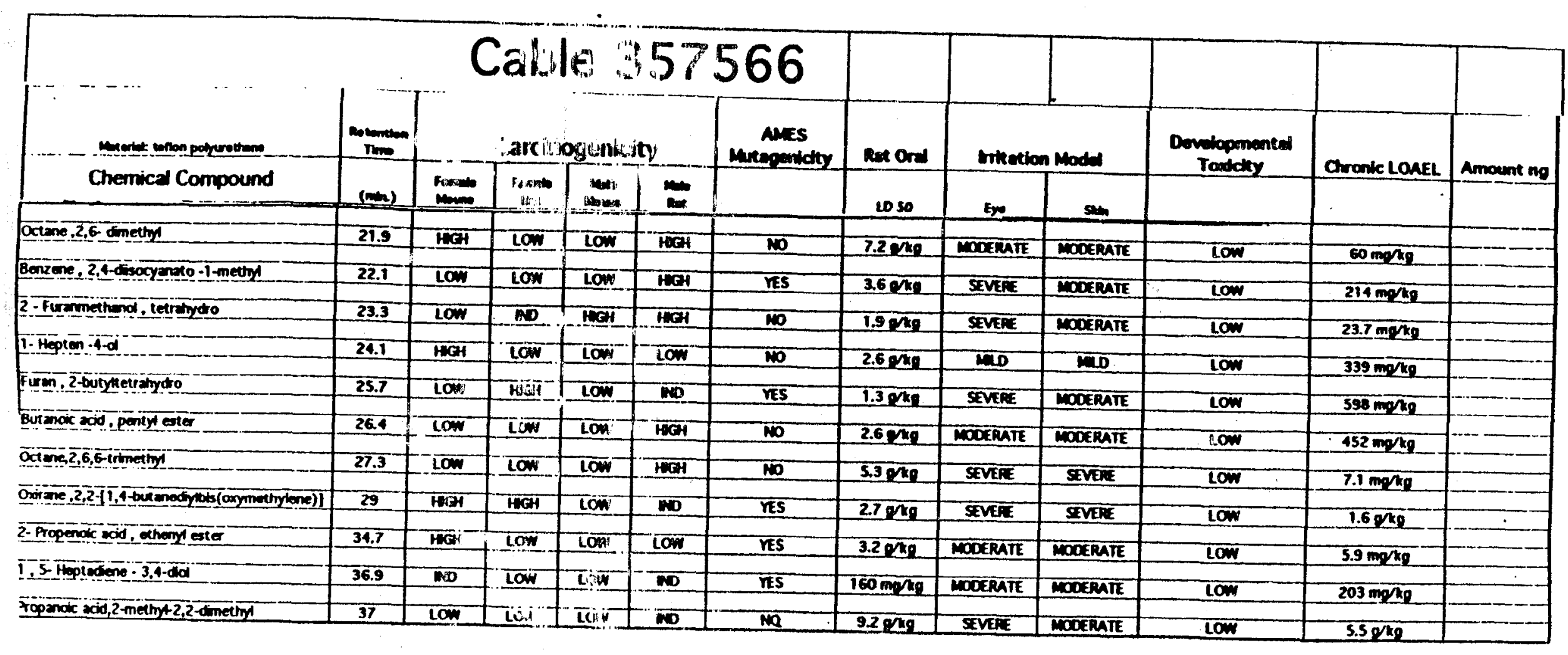


TIC of alqTefpour.d

Abundance

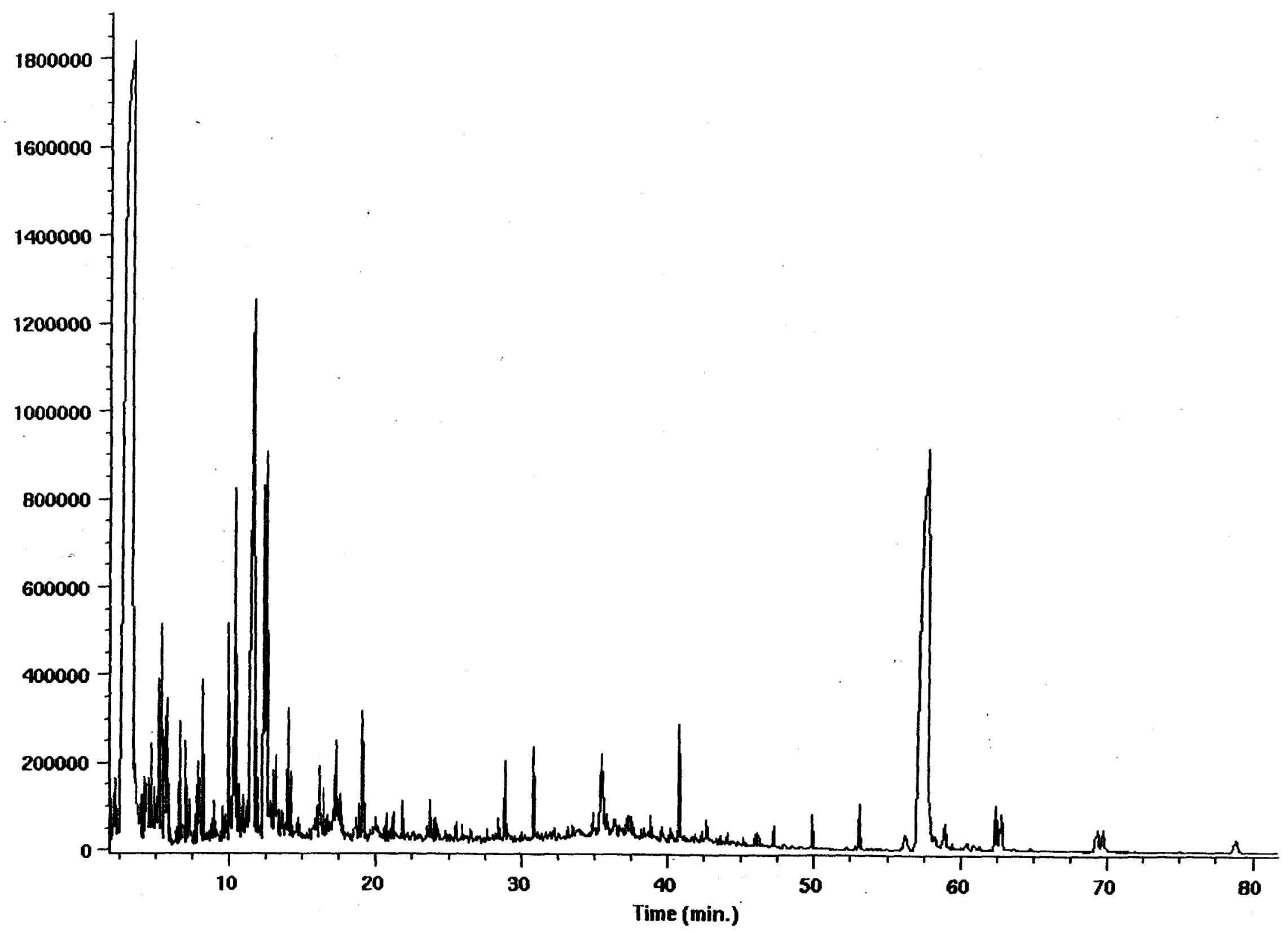

